\title{
An Amplitude-controllable 3-D Hyperchaotic Map with Homogenous Multistability
}

\section{Xuejiao Zhou}

Nanjing University of Information Science and Technology

Chunbiao Li ( $\square$ chunbiaolee@nuist.edu.cn )

Nanjing University of Information Science and Technology https://orcid.org/0000-0002-9932-0914

\section{Yongxin Li}

Nanjing University of Information Science and Technology

Xu Lu

Nanjing University of Information Science and Technology

Tengfei Lei

Qilu Institute of Technology

\section{Research Article}

Keywords: Hyperchaotic map, amplitude control, homogenous multistability

Posted Date: April 13th, 2021

DOI: https://doi.org/10.21203/rs.3.rs-411243/v1

License: (c) (1) This work is licensed under a Creative Commons Attribution 4.0 International License.

Read Full License

Version of Record: A version of this preprint was published at Nonlinear Dynamics on July 8th, 2021. See the published version at https://doi.org/10.1007/s11071-021-06654-2. 


\title{
An amplitude-controllable 3-D hyperchaotic map with homogenous
}

\author{
multistability \\ Xuejiao Zhou ${ }^{1}$, Chunbiao $\mathrm{Li}^{1,2,3}$ a), Yongxin $\mathrm{Li}^{1}, \mathrm{Xu} \mathrm{Lu}^{1}$, Tengfei Lei ${ }^{3}$ \\ ${ }^{1}$ Jiangsu Collaborative Innovation Center of Atmospheric Environment and Equipment \\ Technology (CICAEET), Nanjing University of Information Science \& Technology, \\ Nanjing 210044, China \\ ${ }^{2}$ School of Artificial Intelligence, Nanjing University of Information Science \& \\ Technology, Nanjing 210044, China \\ ${ }^{3}$ Collaborative Innovation Center of Memristive Computing Application (CICMCA), \\ Qilu Institute of Technology, Jinan 250200, China
}

\begin{abstract}
By introducing a sinusoidal function into a three-dimensional map, a hyperchaotic map with three positive Lyapunov exponents is derived. The system has two amplitude controllers, a total controller, and a partial controller. The hyperchaotic map shares a unique structure of two-leaf and three-leaf attractors under united Lyapunov exponents. Furthermore, homogenous multistability is found in the 3-D map, where the initial data determine the attractor structure combined with the distance between any two leaves. Experimental hardware based on STM32 is built to prove the numerical findings. The hyperchaotic map is introduced for color image encryption. The analysis of key space, histogram, information entropy, correlation, and anti-noise infection shows its powerful performance in encryption and security.
\end{abstract}

Keywords: Hyperchaotic map; amplitude control; homogenous multistability

\section{Introduction}

Chaos plays an important role in image encryption [1-5], secure communication [6-8], computer engineering [9-10], and even other fields[11-12]. The discrete chaotic map shows its potential power as a continuous chaotic system. Amplitude control and polarity adjustment of the chaotic sequence obtain much flexibility for chaos-based

a) Corresponding author. E-mail address: goontry@126.com; chunbiaolee@nuist.edu.cn 
applications and therefore introduce much more value in information engineering [13-17]. Although the amplitude and offset control of continuous chaotic systems have been systematically explored and reported [18-19], the geometric control of discrete chaotic maps is still in the beginning stages. Trigonometric function nonlinearity brings great convenience for chaos production [20], but it also intensifies the difficulty of amplitude control and poses a great risk for multistability[21-25]. It is of great theoretical significance and engineering values to design chaotic maps based on trigonometric function nonlinearity and study their controllability. A couple of 2-D chaotic maps with trigonometric functions have been studied [26-29], with abundant dynamics for multistability. However, we cannot find any single knob for amplitude control. Amplitude control along with offset boosting seems to be well addressed in continuous chaotic systems [30], but all chaotic systems ignore the nonlinearity of the trigonometric function. Continuous chaotic systems with trigonometric functions have been investigated, mainly focusing on attractor self-reproduction [31-32] or attractor growing[33-34] rather than amplitude control. Amplitude control in chaotic maps seems much more difficult to design since the coefficient for variable rescaling has a broader distribution on the right side of the map; therefore, the control of chaotic maps has not received enough attention in the field of nonlinear dynamics. Additionally, homogenous multistability plays an important role in amplitude control, where an initial condition can be selected for easy variable rescaling.

Aiming to design a chaotic map with amplitude control and rich dynamics, in this paper, a three-dimensional (3-D) hyperchaotic map is created by resorting to sinusoidal feedback. As a result, the system is gifted with two independent knobs for total and partial amplitude control. In section 2, the hyperchaotic model is constructed, and the dynamic development is discussed, including fixed points and bifurcation analysis. In section 3 the principle of amplitude control is explained. In section 4, the special homogenous multistability is demonstrated, where 2-leaf and 3-leaf attractors are selected by the initial condition under a set of unified Lyapunov exponents. In section 5, a digital platform based on STM32 is set up for experimental verification. 
In section 6, the hyperchaotic sequence is applied to image encryption, where the performance of encryption affected by amplitude control and homogenous multistability is exhaustively explored. The conclusions are presented in the last section.

\section{A 3-d hyperchaotic map and its basic dynamics}

\subsection{System model}

Based on the continuous jerk system, a new 3-D discrete chaotic map is derived by introducing a sinusoidal function,

$$
\left\{\begin{array}{l}
x_{n+1}=x_{n}+a x_{n} \sin \left(b y_{n}\right) \\
y_{n+1}=z_{n} \\
z_{n+1}=c x_{n}-y_{n}-z_{n}
\end{array}\right.
$$

where $x_{n}, y_{n}, z_{n}(n=0,1,2, \ldots)$ are discrete sequences, and system parameters $a, b, c$ are not equal to 0 .

The fixed points $S=\left(x^{*}, y^{*}, z^{*}\right)$ of the system (1) is solved by the formula (2),

$$
\left\{\begin{array}{l}
x^{*}=x^{*}+a x^{*} \sin \left(b y^{*}\right) \\
y^{*}=z^{*} \\
z^{*}=c x^{*}-y^{*}-z^{*}
\end{array}\right.
$$

Therefore, the fixed points are shown as,

$$
S=\left(x^{*}, y^{*}, z^{*}\right)=\left(\frac{3 m \pi}{b c}, \frac{m \pi}{b}, \frac{m \pi}{b}\right)
$$

where $m$ is an integer.

When $a=0.9, b=1$, and $c=-2$, the Jacobian matrix of the discrete system (1) at the fixed point $\left(x^{*}, y^{*}, z^{*}\right)=(-3 m \pi / 2, m \pi, m \pi)(m=0,1,2, \ldots)$ can be described as

$$
J=\left[\begin{array}{ccc}
1 & -1.35 m \pi \cos (m \pi) & 0 \\
0 & 0 & 1 \\
-2 & -1 & -1
\end{array}\right]
$$

The Jacobian characteristic equation satisfies $\lambda^{3}=1+2.7 m \pi \cos (m \pi)(m=0,1,2, \ldots)$. For $m=0,1,2 \ldots,|1+2.7 m \pi \cos (m \pi)| \geq 1$, so $\left|\lambda_{1,2}\right|<1$, it cannot be satisfied at the same time. The system has unstable fixed points. 
When $a=0.9, b=1, c=-2$, and IC $=(-0.1,-0.1,0.1)$, the map (1) exhibits hyperchaos with three positive Lyapunov exponents, $\mathrm{LE}_{1}=0.095, \mathrm{LE}_{2}=0.057$, and $\mathrm{LE}_{3}=0.011$. The corresponding hyperchaotic sequence and attractors are shown in Fig. 1.
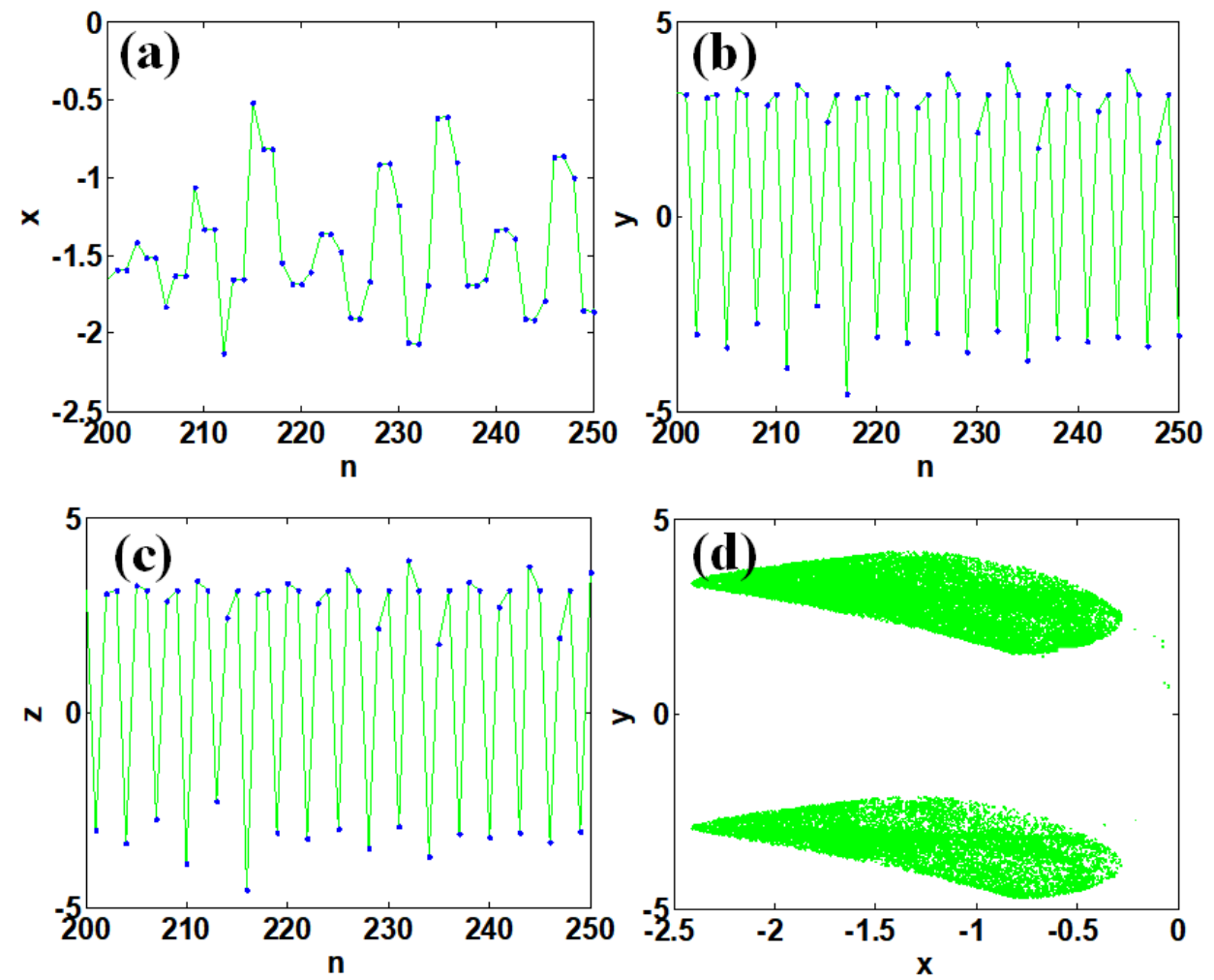

Fig. 1. Hyperchaotic map (1) with $a=0.9, b=1, c=-2$, IC $=(-0.1,-0.1,0.1)$ : (a) x(n), (b) $y(n),(c) z(n)$, and (d) phase portrait in the $x-y$ plane.

\subsection{Bifurcation analysis}

For map (1) with $b=1$ and $c=-2, \mathrm{IC}=(-0.1,-0.1,0.1)$, and parameter $a$ varies in $[0.85,0.95]$, the dynamical behavior can be indicated by the Lyapunov exponent spectrum and corresponding bifurcation as displayed in Fig. 2. When $a \in[0.85,0.862]$ and $a=0.95$, all Lyapunov exponents are negative, and the system is periodic. The typical $x-y$ plane phase portraits are shown in Fig.3(a) and (d). When $a \in[0.863$, 0.868], system (1) has one positive Lyapunov exponent showing its chaotic state. The typical $x-y$ plane phase portraits are shown in Fig. 3(b). When $a \in[0.9,0.94]$ and $a \in$ $[1,1.02]$, three Lyapunov exponents are all positive indicating that system (1) is hyperchaotic, corresponding phase portraits are demonstrated in Fig. 3(c). 

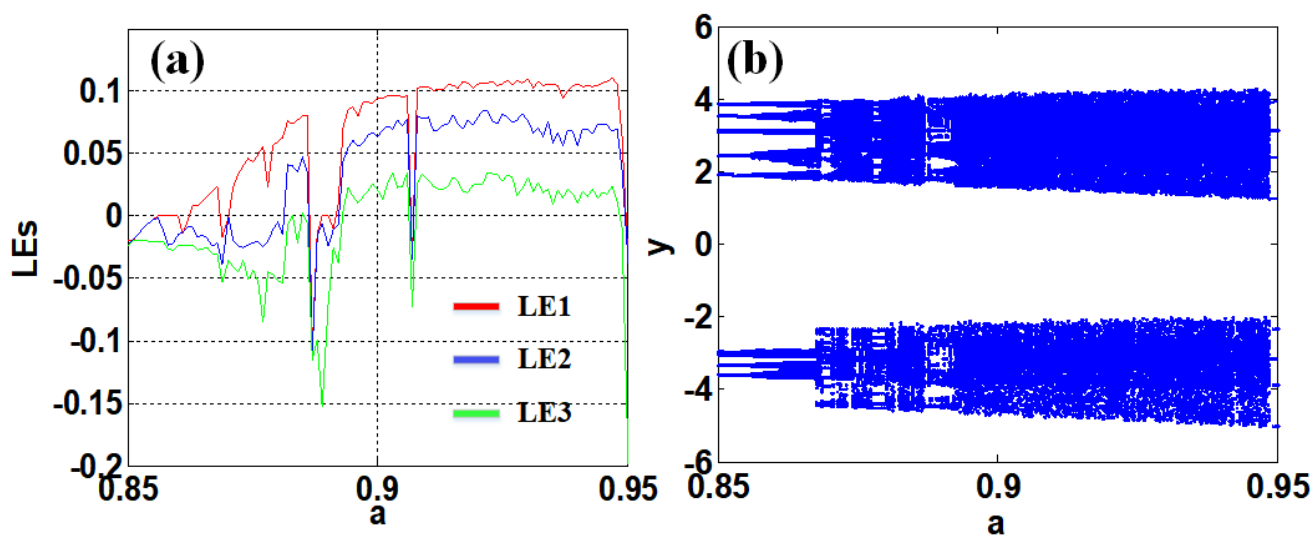

Fig. 2. Dynamical analysis of map (1) when $b=1, c=-2$, and IC $=(-0.1,-0.1,0.1)$ : (a) Lyapunov exponent spectrum and (b) bifurcation evolvement.
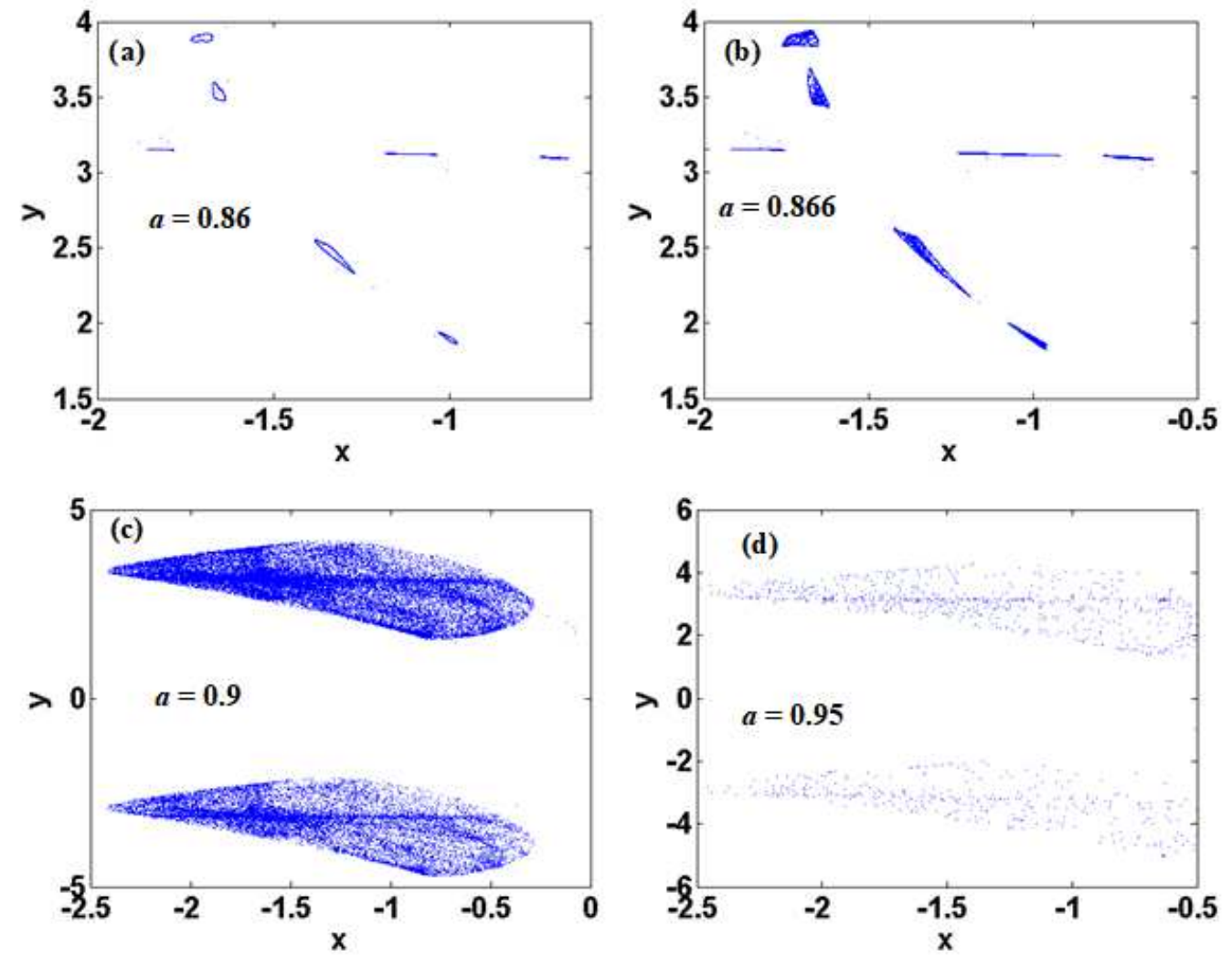

Fig. 3. Typical phase portraits of map (1) with $b=1, c=-2$, IC $=(-0.1,-0.1,0.1)$ with given parameter of $a$ : (a) $a=0.86$, (b) $a=0.866$, (c) $a=0.9$, and (d) $a=0.95$.

Table 1 Les and attractor types for different values of $a$ and fixed IC $=(-0.1$, $-0.1,0.1)$, parameters $b=-1, c=-2$.

\begin{tabular}{|c|c|c|}
\hline$A$ & Attractors & Lyapunov exponents \\
\hline$a=0.86$ & Periodic & $(0,-0.1477,-0.02449)$ \\
\hline$a=0.866$ & Chaotic & $(0.01503,-0.1773,-0.02504)$ \\
\hline
\end{tabular}




\begin{tabular}{|c|c|c|}
\hline$a=0.9$ & Hyperchaotic & $(0.09541,0.05661,0.01103)$ \\
\hline$a=0.95$ & Periodic & $(0,-0.1477,-0.02449)$ \\
\hline
\end{tabular}

\section{Amplitude control analysis}

\subsection{Total amplitude control}

Theorem 1: In system (1), parameter $b$ is a total amplitude controller ${ }^{16}$.

Proof 1: Let $u_{n+1}=x_{n+1} / b, v_{n+1}=y_{n+1} / b$ and $w_{n+1}=z_{n+1} / b$, the resulting system is

$$
\left\{\begin{array}{l}
u_{n+1}=u_{n}+a u_{n} \sin \left(v_{n}\right) \\
v_{n+1}=w_{n} \\
w_{n+1}=c u_{n}-v_{n}-w_{n}
\end{array}\right.
$$

which is identical to the equation (1) with $b=1$. Therefore, the parameter $b$ adjusts the amplitude of all sequences of $x, y$ and $z$ according to $1 / b$, which means that $b$ is a total amplitude controller.

Therefore, parameter $b$ controls the amplitude of all variables. The parameter $b$ is a nonbifurcation parameter. As shown in Fig. 4, the amplitudes of all variables are controlled under different $b$. In system (1), $a=0.9, c=-2$, and IC $=(-0.1,-0.1,0.1)$. When $b$ takes different values, the phase portraits are plotted in Fig. 4.
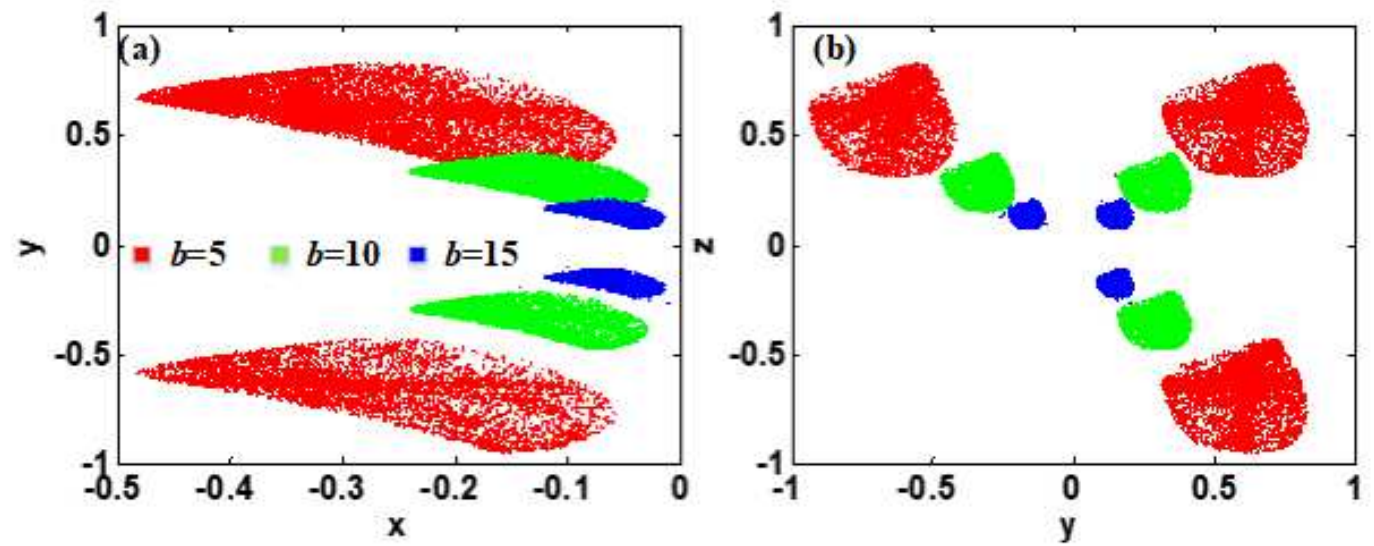

Fig. 4. Two views of the phase portraits of map (1) with $\mathrm{a}=0.9, \mathrm{c}=-2$, and $\mathrm{IC}=(-0.1$, $-0.1,0.1$ ), where parameter $\mathrm{b}$ takes different values: (a) $\mathrm{x}-\mathrm{y}$ plane and (b) $\mathrm{y}-\mathrm{z}$ plane.

Further verification can be seen in Fig. 5, where the averages of the absolute values of 
$x, y$, and $z$ increase with the parameter $b$ but almost without revising the Lyapunov exponents. It also further proves that the frequency of the sequence in the system (1) is not changed by parameter $b$.
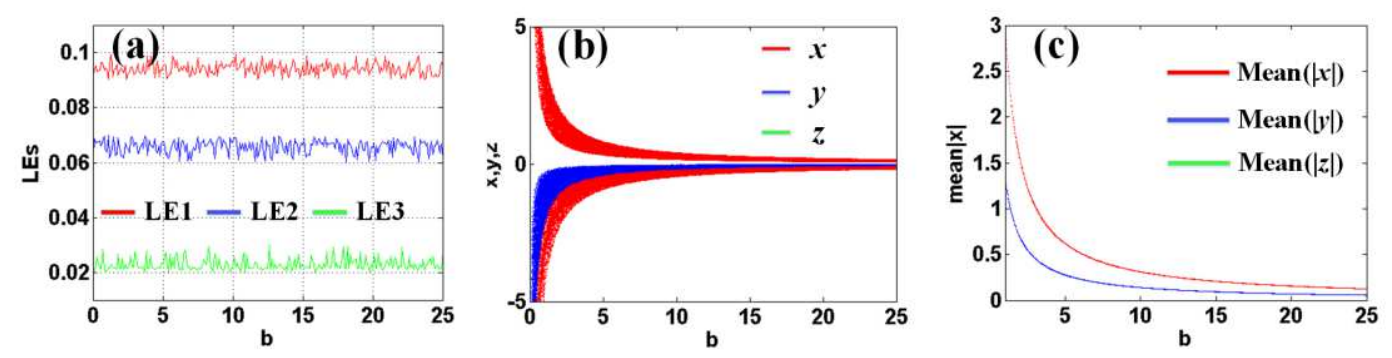

Fig. 5. Amplitude control in system (1) when $a=0.9, c=-2$, and IC $=(-0.1,-0.1,0.1)$ : (a) almost invariable Lyapunov exponents with the evolvement of parameter $b$, (b) bifurcation diagram, and (c) the average of all absolute values of the sequences.

\subsection{Partial amplitude control}

Theorem 2: The parameter $c$ in the system (1) is a partial amplitude controller ${ }^{16}$.

\section{Proof 2:}

Let $u_{n+1}=x_{n+1} / c, v_{n+1}=y_{n+1}$, and $w_{n+1}=z_{n+1}$; the resulting system is

$$
\left\{\begin{array}{l}
u_{n+1}=u_{n}+a u_{n} \sin \left(b v_{n}\right) \\
v_{n+1}=w_{n} \\
w_{n+1}=u_{n}-v_{n}-w_{n}
\end{array}\right.
$$

Therefore, the parameter $c$ controls the amplitude of the sequence of $x$ according to $1 /$ $c$ without changing the other two series of $y$ and $z$. Therefore, parameter $c$ is a nonbifurcation partial amplitude controller for the variable $x$.

As shown in Fig. 6(b), the amplitude of the $x$ signal is controlled under different $c$. When $c=-2$, the amplitude of $x$ is large, and when $c=-20$, the amplitude is greatly reduced. Note that here, the amplitude controller $c$ is derived differently from the case in a continuous system. Further verification can be seen in Fig. 6(c), where the average of the $|x(n)|$ increases with parameter $c$ but almost without revising the Lyapunov exponents. This also further proves that the frequency of the sequence in the system (1) is not changed by parameter $c$. 

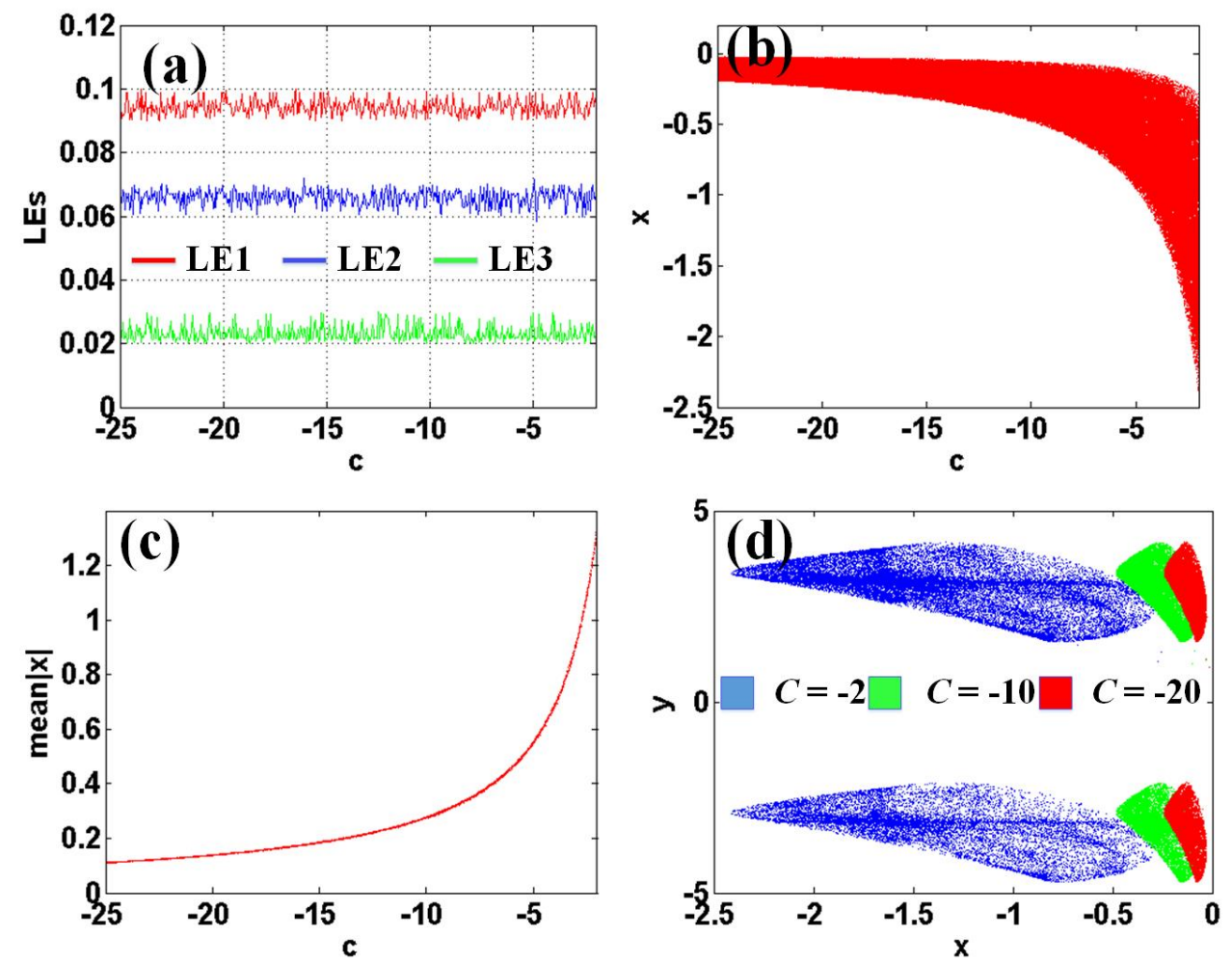

Fig. 6. Amplitude-controlled sequence $x(\mathrm{n})$ in system (1) with $a=0.9, b=1$, and $\mathrm{IC}=$ $(-0.1,-0.1,0.1)$ : (a) Lyapunov exponent spectrum, (b) bifurcation diagram of $x(\mathrm{n})$, (c) average value of $|x(\mathrm{n})|$, and (d) rescaled phase portraits under different $c$.

\section{Homogeneous multistability}

Different initial conditions in chaotic systems may lead to different attractors of the same shape (sometimes with different amplitudes and/or frequencies or offsets). This special multistability is defined as homogeneous multistability.

To show the complex dynamics of system (1) with infinitely many attractors, a bifurcation diagram of the initial value is drawn in Fig. 7, with $a=0.9, b=1, c=-1$, and $\mathrm{IC}=\left(-1, y_{0}, 1\right)$. It proves that the system has multistability. 

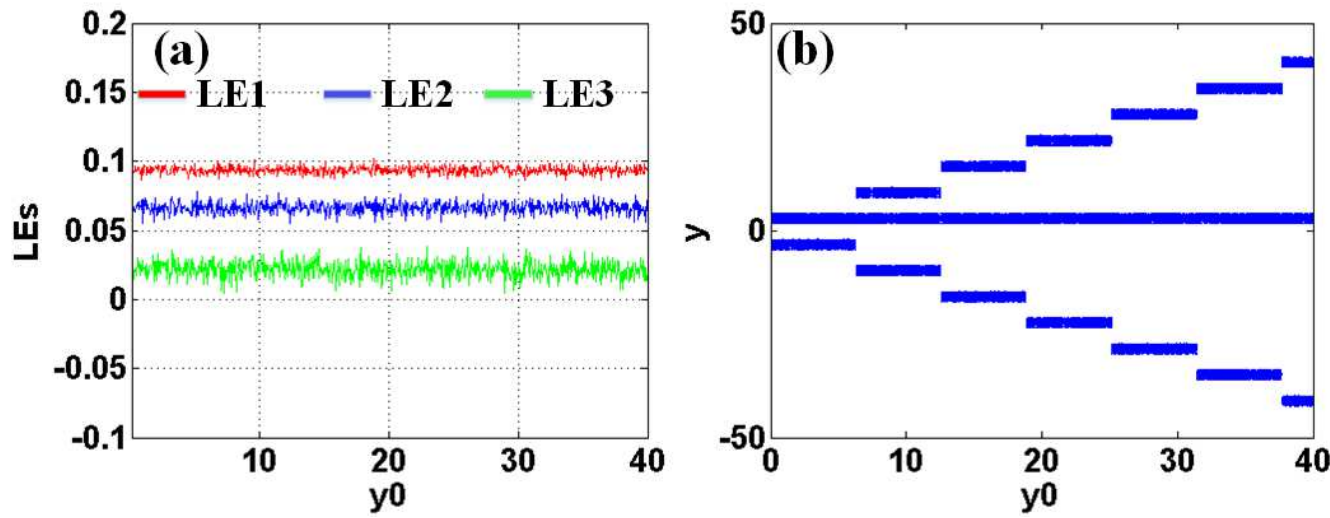

Fig. 7. Dynamical transition of map (1) at $a=0.9, b=1, c=-1$, and IC $=(-1, \mathrm{y} 0,1)$ : (a) Lyapunov exponent spectrum and (b) bifurcation controlled by the initial condition.
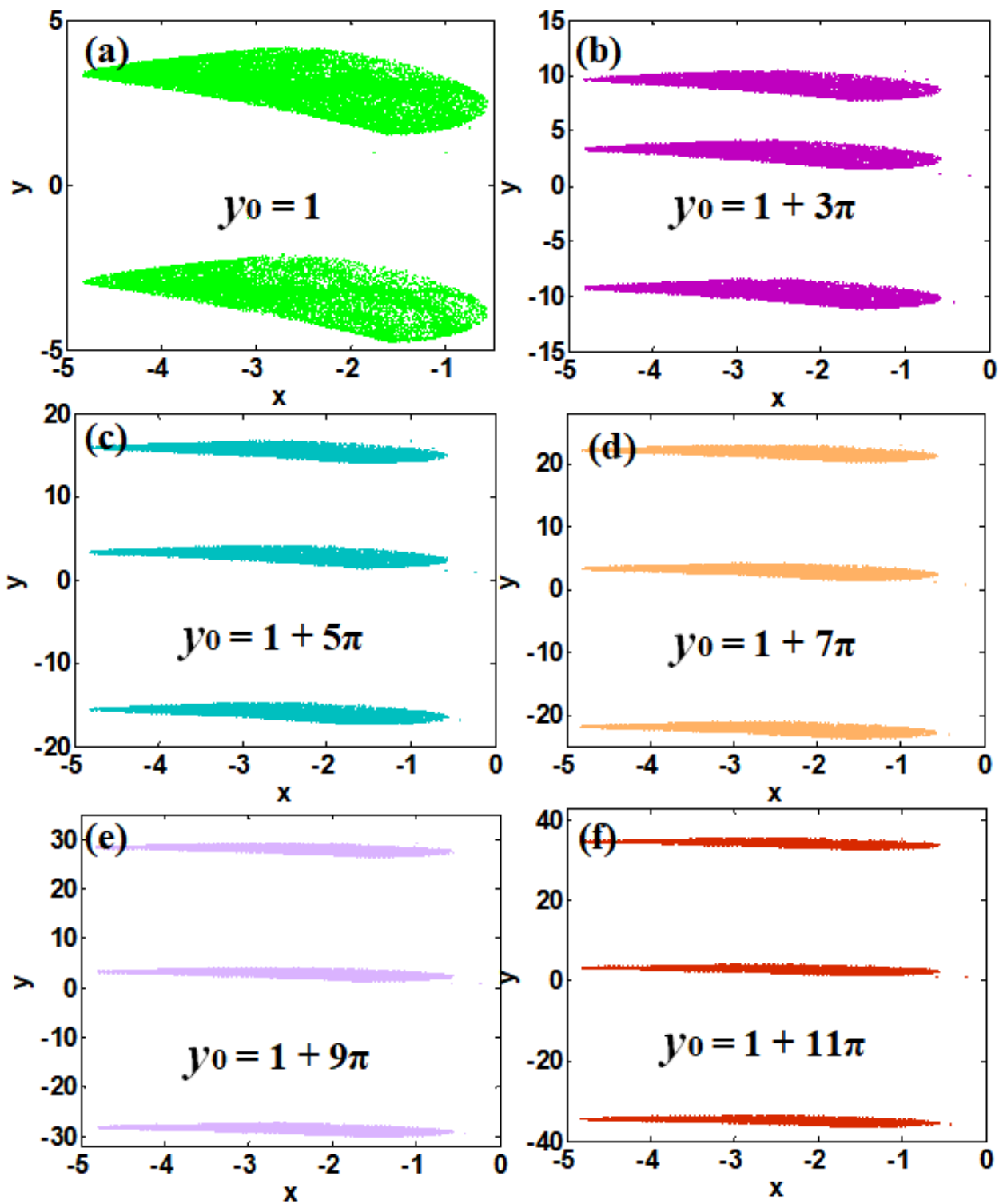

Fig. 8. Rescaled coexisting two-leaf and three-leaf attractors from map (1) with $a=$ $0.9, b=1, c=-1, \mathrm{IC}=\left(-1, y_{0}, 1\right)$ under the different initial conditions of $y_{0}$. 
Rescaled coexisting two-leaf and three-leaf attractors are plotted in Fig. 8. System (1) has infinite coexisting attractors with unified Lyapunov exponents. System (1) parameters are set to $a=0.9, b=1, c=-1$, and $\mathrm{IC}=\left(-1, y_{0}, 1\right)$. The system orbits under different initial conditions of $y$ are given in Fig. 8 respectively.

In map (1), when $a=0.9, b=1, c=-1$, and IC $=\left(-1,-1, z_{0}\right)$, the initial value of the Lyapunov exponent spectrum and its bifurcation of the $y$ signal are plotted in Fig. 9. It is interesting that here a new regime of homogenous multistability is found. Rescaled coexisting two-leaf and three-leaf hyperchaotic attractors share the same set of unified Lyapunov exponents. Moreover, the discrete periodic points are scattered between any two hyperchaotic states. To the best of our knowledge, this is a brand new phenomenon in nonlinear mapping. Also with the increase of $z_{0}$ in the 3-D map, two-leaf and three-leaf attractors get emerged. More strikingly, the initial data also modifies the distance between any two leaves in a positive correlation. Figure 10 shows the typical coexisting phase portraits of the system (1) accordingly.
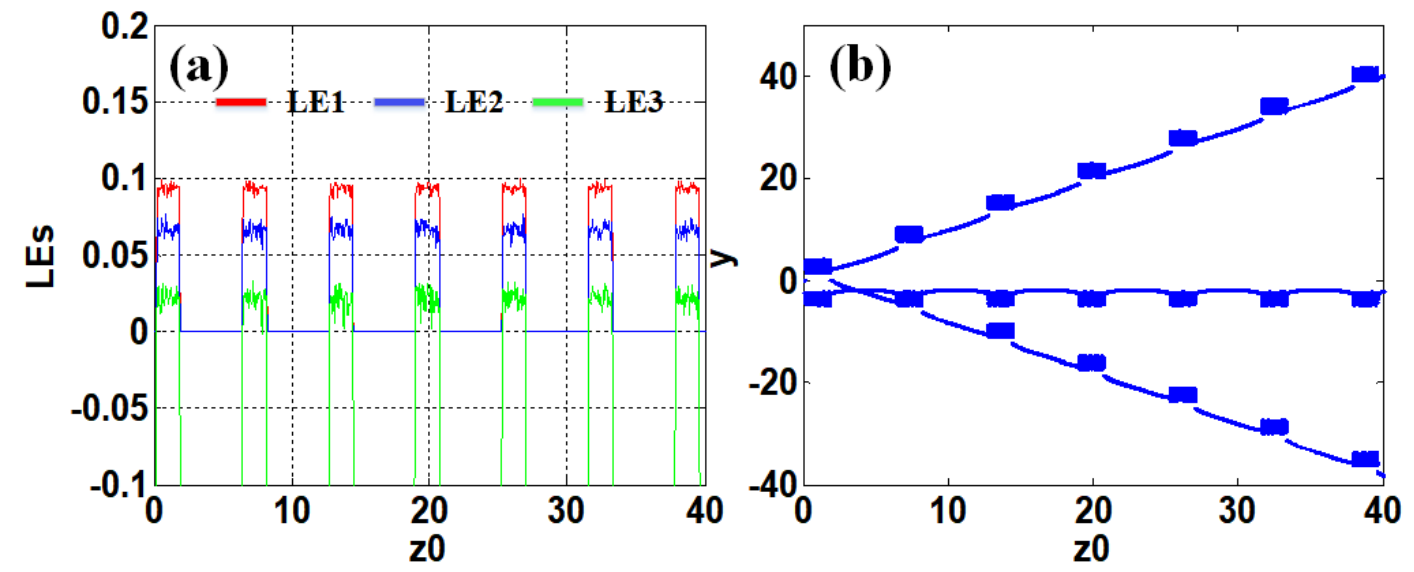

Fig. 9. Dynamical development of map (1) at $a=0.9, b=1, c=-1$, and IC $=(-1,-1$, $\left.z_{0}\right)$ : (a) Lyapunov exponent spectrum and rescaled bifurcation. 

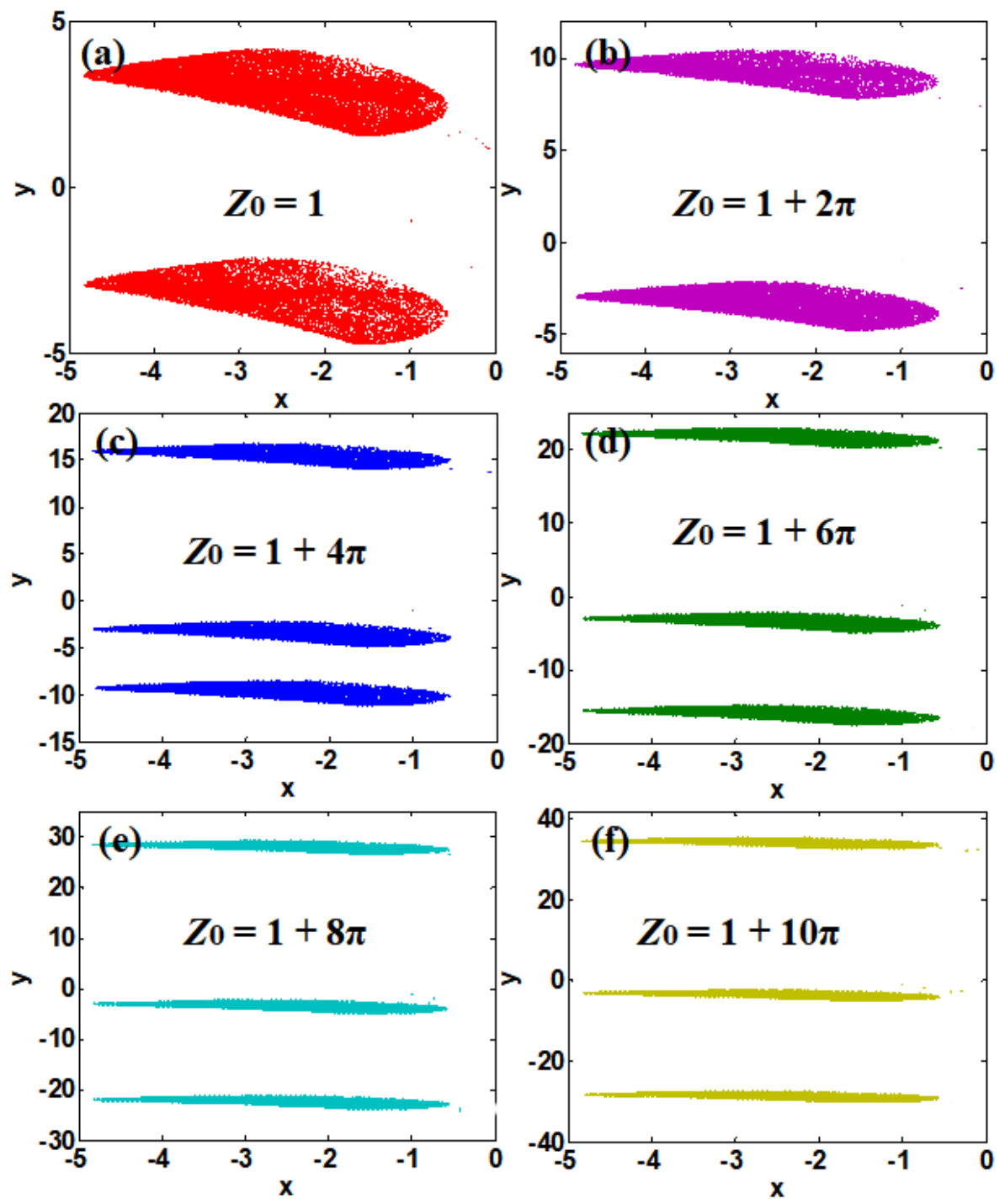

Fig. 10. Rescaled coexisting two-leaf and three-leaf of map (1) with $a=0.9, b=1, c=$ $-1, \mathrm{IC}=\left(-1,-1, z_{0}\right)$ under different initial conditions of $z$.

\section{Hardware implementation based on stm32}

An experimental test can be given for further demonstration. In this work, the MCU development suite was used for experimental exploration. Here the electronic platform and components mainly include STM32F103 MCU and TLV5618, 12-bit DAC modules. To meet the requirement of the precision, here we select $\Delta T=0.001$ and the TLV5618 is applied for giving two separate sequences. The phase trajectories of system (1) are captured in the oscilloscope which is displayed in Fig.11. The experimental process is shown in Fig.12. 

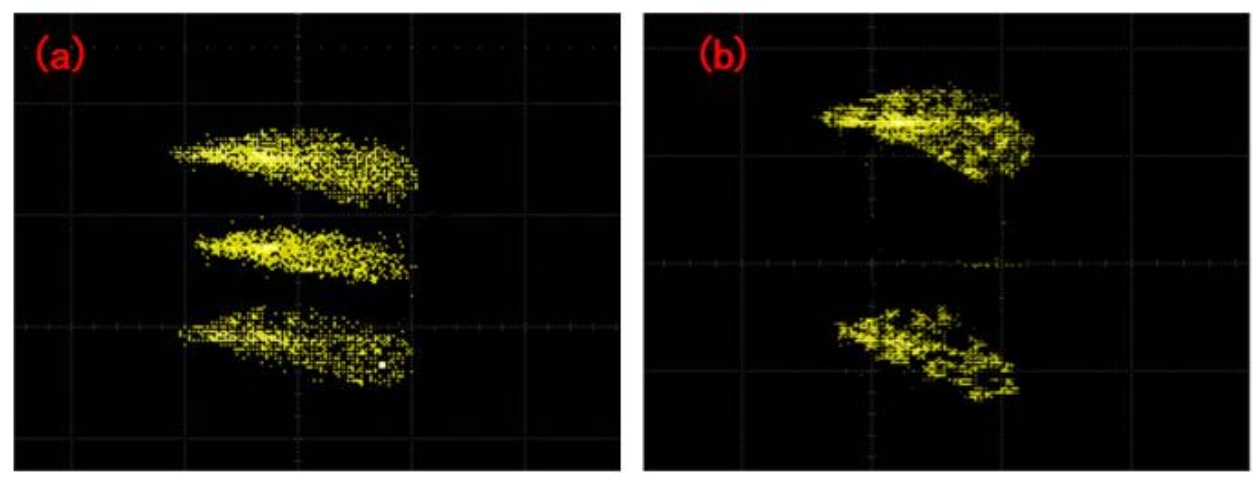

Fig. 11 Phase portraits of map (1) displayed in the oscilloscope when $a=0.9, b=1, c$ $=-1$, and $\mathrm{IC}=\left(-1, y_{0}, 1\right):$ (a) $y_{0}=1$ and (b) $y_{0}=-1$.

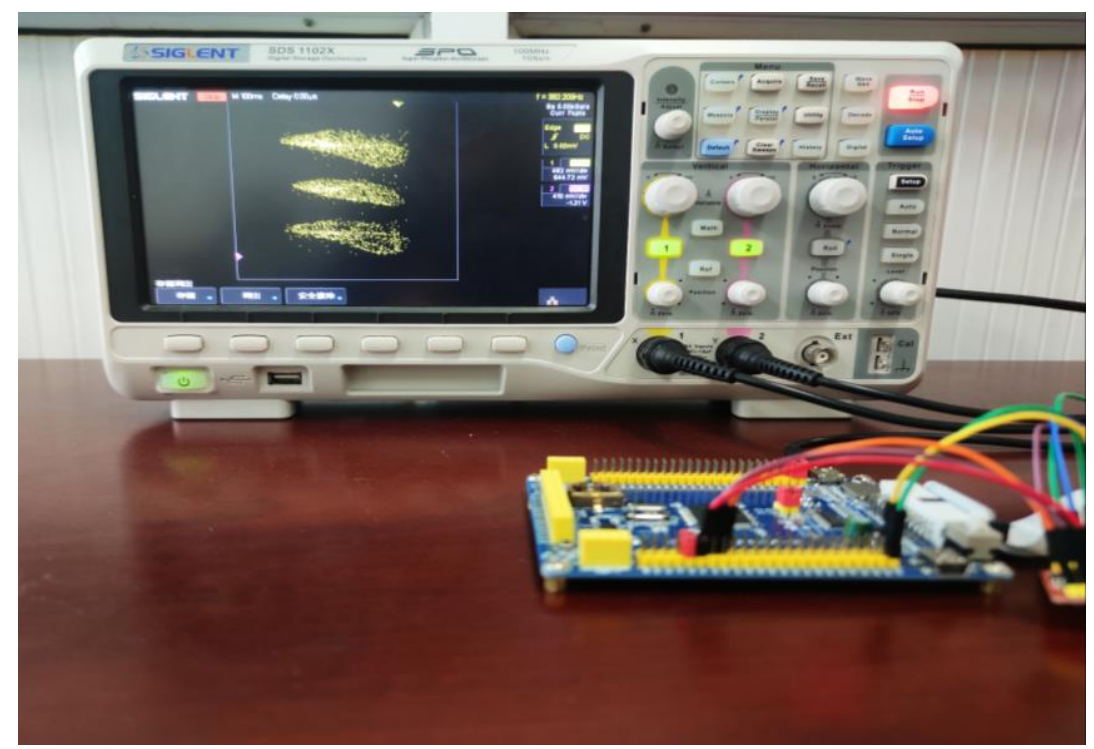

Fig. 12. Hardware equipment of the experimental device

\section{Application of the hyperchaotic map in image encryption}

Hyperchaotic systems provide a larger key space and higher complexity and therefore become more applicable for encryption and secure communication. In the following, the image encryption is studied based on the above proposed hyperchaotic map. Furthermore, the amplitude control and coexisting two-leaf or three-leaf hyperchaotic sequences are applied as shown later that in fact the information entropy of the three-channel encrypted image does not revised dramatically.

\subsection{Algorithm analysis}

Here the DNA coding is applied for image encryption based on the hyperchaotic map 
(1). Compared with other algorithms, the encryption key space becomes larger with stronger sensitivity, showing robustness to attacks. The main encryption flowchart is shown in Fig. 13: first, the image is encrypted by a logistic map, then encrypted by the hyperchaotic sequence combining with the DNA coding. The encryption process is shown in Fig. 13.

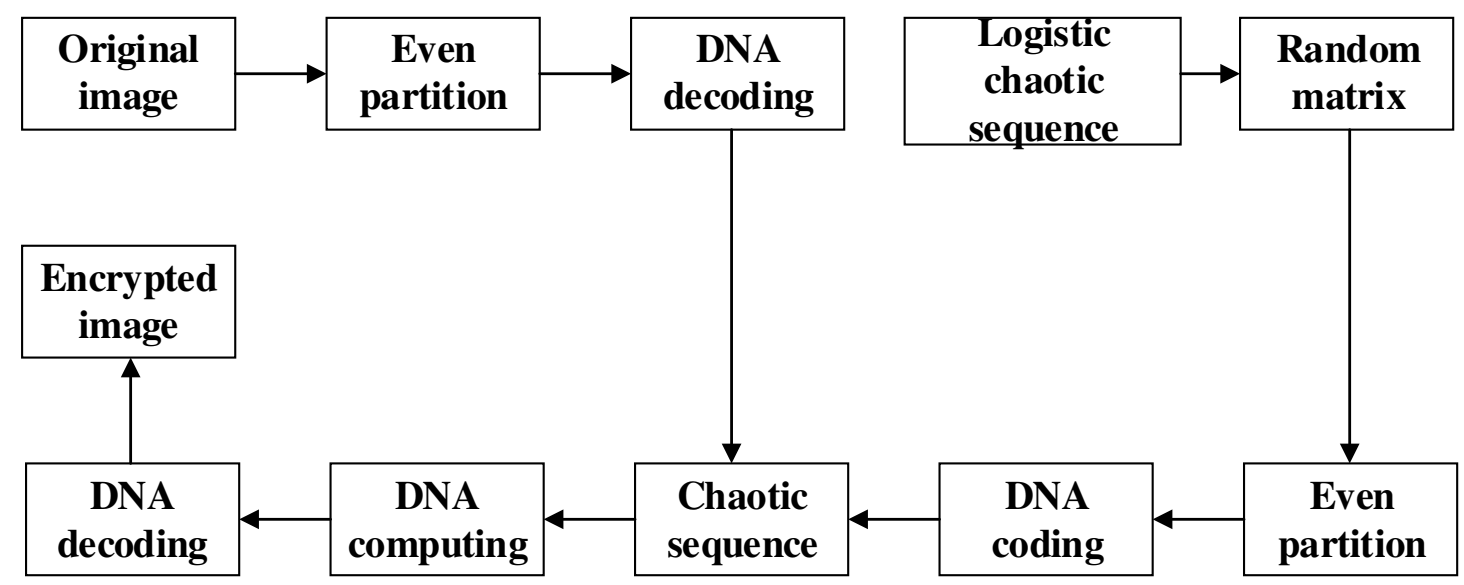

Fig. 13. Encryption flowchart.

The procedure is described as the following:

Step 1: Fill the horizontal and vertical of the picture with $M_{0}=0$ and $N_{0}=0$ so that they are divisible by the block size $t$.

Step 2: Given the initial condition of $x_{0}$ and the variable $\mu$ of the logistic map, discrete signals $\left\{P_{\mathrm{i}}\right\}(\mathrm{i}=2001,2002, \ldots, \mathrm{M} \times \mathrm{N}+2000)$ with the length of $\mathrm{M} \times \mathrm{N}+2000$ is obtained.

Step 3: All the numbers in the discrete signals are transformed to $[0,255]$ and then randomly formed into a 2-D matrix $\mathrm{R}$ of $M \times N$.

Step 4: When we take $t=4$, the size of every block of the matrix $\mathrm{R}$ and the image is set to $t \times t$.

Step 5: Given the initial conditions $\mathrm{X}_{0}, \mathrm{Y}_{0}, \mathrm{Z}_{0}$ and the values of $a$ and $b$ in system (1), three discrete time series $\left\{x_{\mathrm{i}}\right\},\left\{y_{\mathrm{i}}\right\}$ and $\left\{z_{\mathrm{i}}\right\}$ can be obtained $(\mathrm{i}=4002,4003, \ldots$, $4001+M / t+N / t)$.

Step 6: The DNA coding pattern of every image matrix and a stochastic matrix is determined by sequences $\left\{x_{\mathrm{i}}\right\}$ and $\left\{y_{\mathrm{i}}\right\}$. The actual conversion process is as follows, 


$$
x_{i}=\bmod \left(\operatorname{round}\left(x_{i} \times 10^{4}\right), 8\right)+1
$$

Transform the grayscale values of all picture elements to the binary numeral and performs DNA coding in $\left\{x_{i}\right\}$ mode.

Step 7: The DNA coding pattern between random matrix and image matrix is determined by $\left\{z_{i}\right\}$. The conversion of $\left\{z_{i}\right\}$ is as follows,

$$
z_{i}=\bmod \left(\operatorname{round}\left(z_{i} \times 10^{4}\right), 4\right)
$$

when $z_{i}=0$, the random matrix block and the corresponding elements in the image matrix block execute DNA addition, and when $z_{i}=1$, subtraction, $z_{i}=2$, exclusive or (XOR), and $z_{i}=3$, the equivalence gate (XNOR) operation.

Step 8: The encryption performance can be optimized by adding a diffusion algorithm. $\left\{z_{i}\right\}$ also determines the relationship between two adjacent image blocks after encryption. In the case of $z_{i}=0$, block $i$ encryption result $c_{i}$ can be expressed as:

$$
c_{i}=c_{i-1}+I_{i}+R_{i}
$$

The decryption process and the encryption process are mutually inverse. The DNA encoding method and random matrix are obtained by the correct key.

\subsection{Chaotic encryption with DNA coding}

Test with a standard RGB image, as displayed in Fig. 14. Given the initial value $x_{0}$ and parameter $\mu$ of the Logistic map are 0.5475 and 3.999 respectively. The system parameter of hyperchaotic map (1) is $a=0.9, b=1, c=-1$, the initial value of the system is $\mathrm{X}_{0}=-1, \mathrm{Y}_{0}=-1, \mathrm{Z}_{0}=1$, and the coding rule of DNA is generated randomly. Table. 2 lists the selected key and the values of the zeroing parameters $M_{0}$ and $N_{0}$ during the encryption process. $k_{1}$ and $k_{2}$ are the average gray levels of the $\mathrm{B}$ channel and $G$ channel in the unencrypted picture, respectively. The encrypted image simulated with Matlab is displayed in Fig. 14(b). The images before and after encryption are completely different. Figure 14(c) is the correctly decrypted picture, which is identical to the unencrypted image. 
Table 2 Algorithm keys.

\begin{tabular}{|c|c|c|c|c|c|}
\hline Key & $\mu$ & $x_{0}$ & $X_{0}$ & $Y_{0}$ & $Z_{0}$ \\
\hline Value & 3.999 & 0.5475 & -1 & -1 & 1 \\
\hline Key & $k_{1}$ & $k_{2}$ & $M_{0}$ & $N_{0}$ & \\
\hline Value & 0.3883 & 0.4134 & 0 & 0 & \\
\hline
\end{tabular}
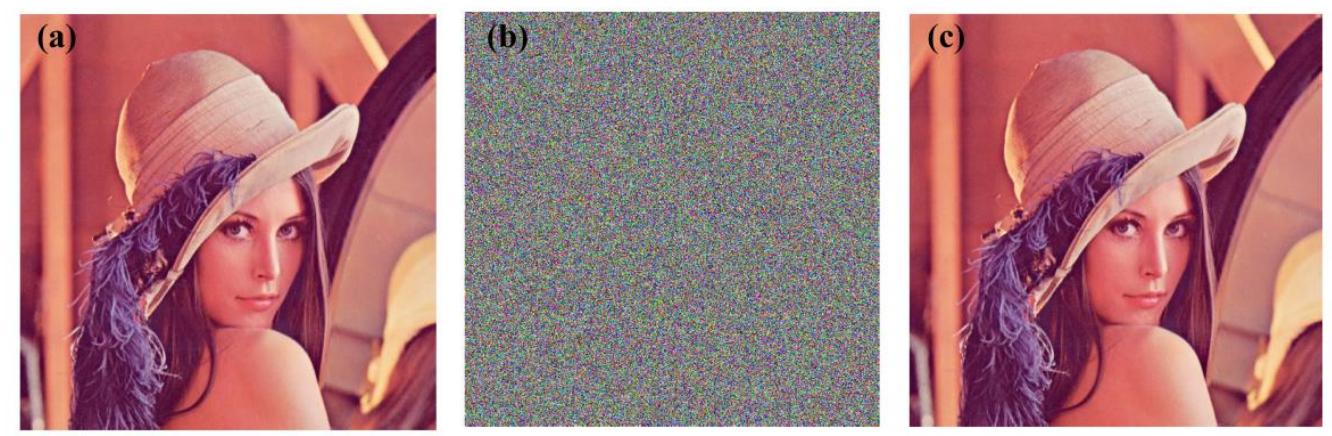

Fig. 14. Encryption experiment image: (a) the unencrypted picture, (b) the encryption picture, and (c) the decryption picture.

\subsection{Safety performance analysis}

Security is the essential requirement of the encryption system. Normally, a sufficiently large key space is necessary for hyperchaotic image encryption systems, as well as reversible encryption processes and powerful anti-attack performance. We have conducted a detailed analysis of the hyperchaotic encryption algorithm from the following five aspects. The specific analysis process is as follows.

\subsubsection{Key space analysis}

An excellent encryption method should make its key space very large to withstand a certain degree of attack. In the encryption algorithm proposed in this work, the initial condition of the 3-D hyperchaotic map is used as the key, and the accuracy up to $10^{-16}$, so the key space can up to $10^{80}$. Besides, the improved logistic chaotic map is used in the key generation algorithm. Its initial values and parameters are also used as a part of the key, so the scheme further expands the key space and can resist the attack more effectively. 


\subsubsection{Histogram analysis}

The RGB components are extracted from the original color picture and its encrypted picture, and their histograms are shown in Fig. 15. It can be easily observed from Fig.15 that the histograms of the R, G, B elements of the picture before and after encryption are completely different.
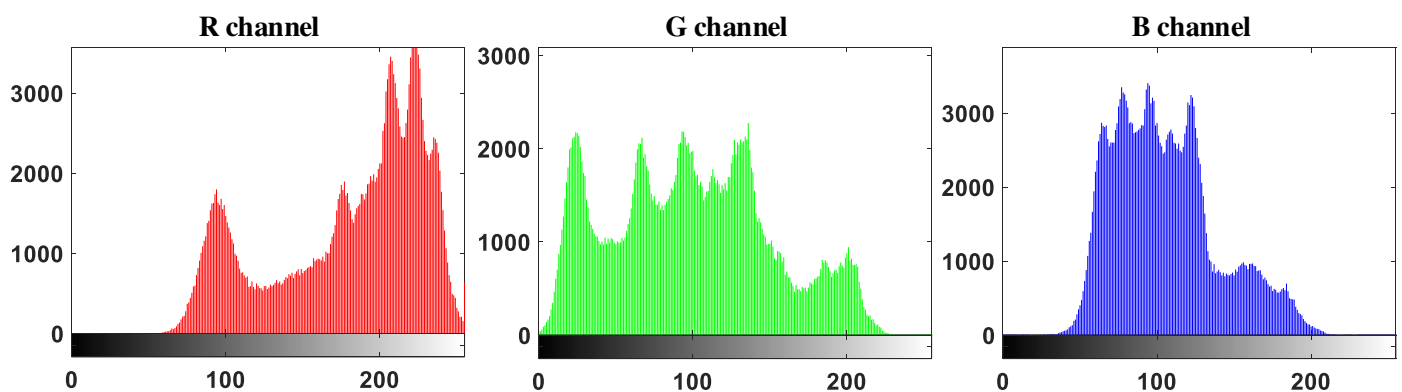

(a)
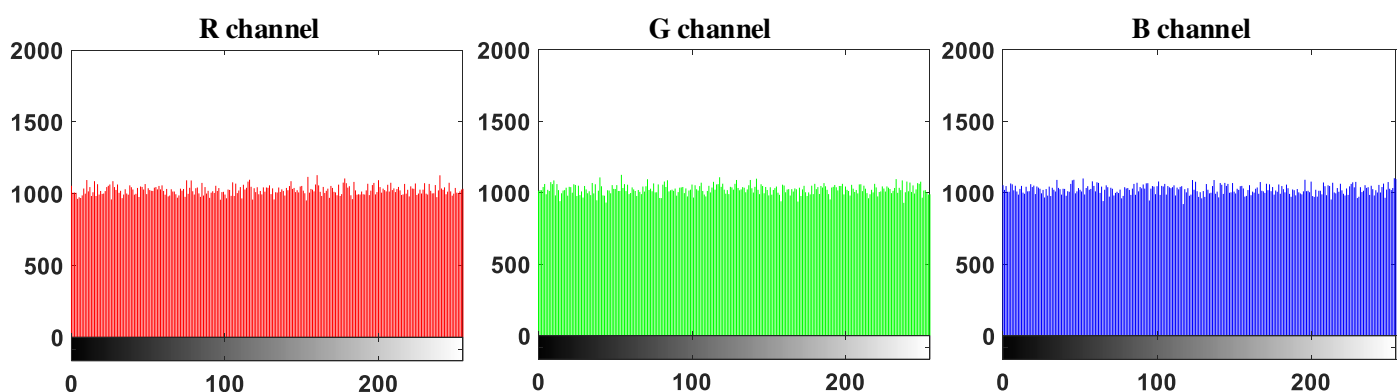

(b)

Fig. 15. Encryption experiment histogram: (a) original picture histogram and (b) encrypted picture histogram.

\subsubsection{Information entropy analysis}

The randomness of image information can be analyzed by information entropy. The size of information entropy is proportional to the strength of randomness. To ensure the random distribution of pixel values, we use the following method to calculate,

$$
H(x)=-\sum_{i=1}^{N} p\left(x_{i}\right) \log _{2} p\left(x_{i}\right)
$$

where $N$ is the number of gray levels, $x_{i}$ is a gray level of the picture, and $P\left(x_{i}\right)$ is the frequency of the grayscale. Generally speaking, the pixel value of a completely random gray image is scattered between $[0,255], p\left(x_{i}\right)=1 / 256, i \in[0,255]$, and the 
entropy is computed to be 8 bits. Therefore, the smaller the difference between the information entropy of the encrypted picture and 8, the better the encoding performance. The information entropy of the three channels of the picture before and after encryption is displayed in Table 3. The information entropy calculated by our method is higher than that in reference ${ }^{25}$ which indicates a good image encryption performance.

Table 3. Information entropy of three channels of the unencrypted picture and encoded images.

\begin{tabular}{|c|c|c|c|}
\hline Image & Red & Green & Blue \\
\hline Original image & 7.2682 & 7.5901 & 6.9951 \\
\hline Encrypted image & 7.9993 & 7.9993 & 7.9994 \\
\hline
\end{tabular}

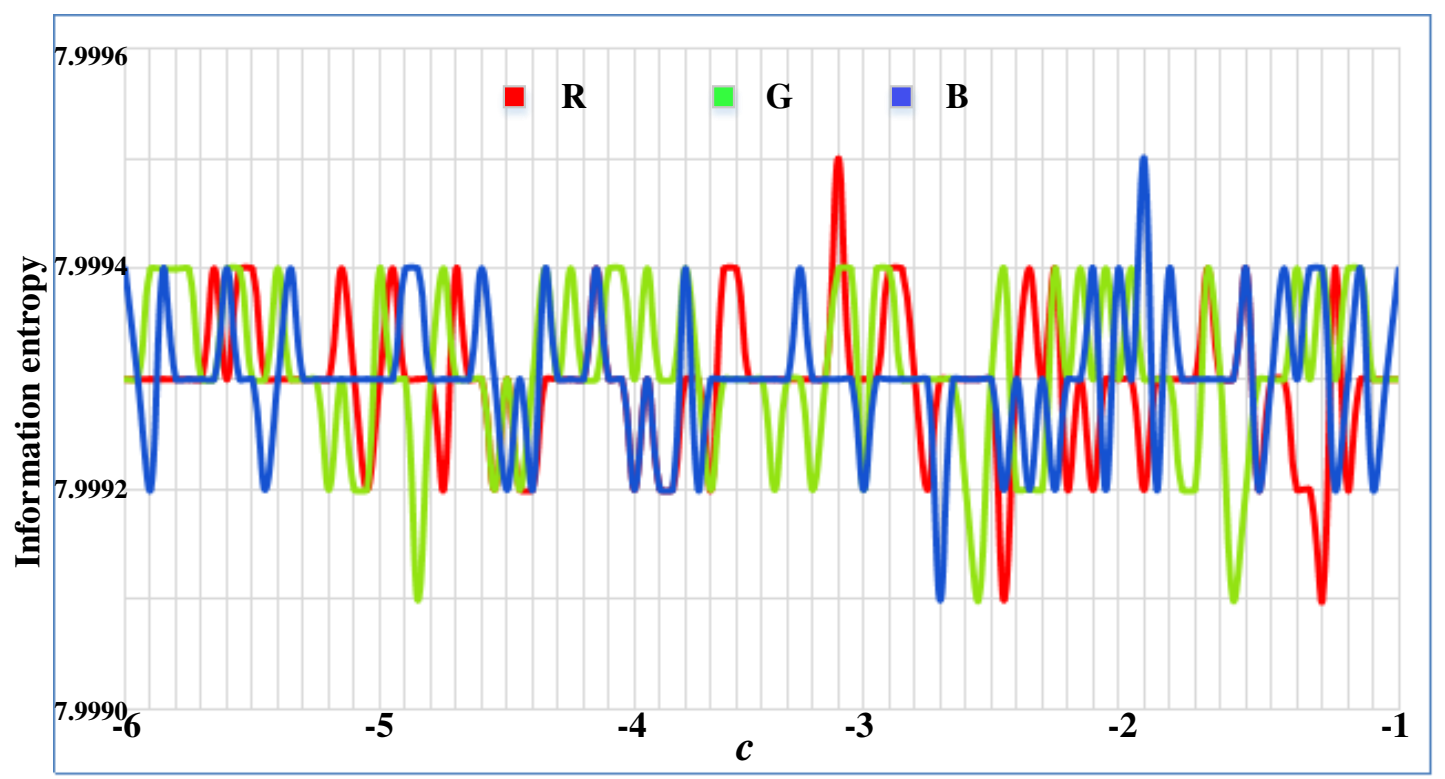

Fig. 16. Information entropy of encrypted image in the R, G, and B channels by the sequences from system (1) with $a=0.9, b=1$, and $\mathrm{IC}=(-1,-1,1)$.

Figure 16 shows the information entropy curves of the encrypted image of the amplitude modulation system at different amplitudes. Figure 17 shows the information entropy of three channels of different multistability encrypted images 


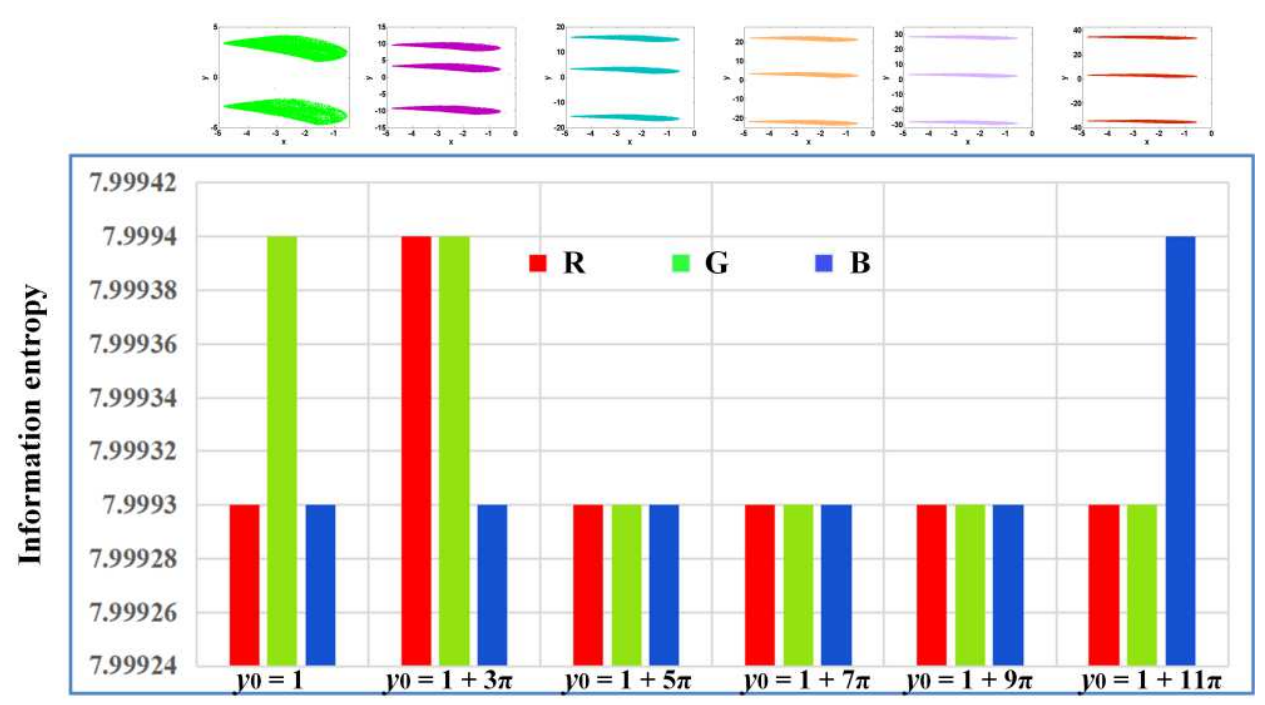

Fig. 17. Information entropy of the three-channel encrypted image by the sequences from system (1) with $a=0.9, b=1, c=-1$ and $\mathrm{IC}=\left(-1, y_{0}, 1\right)$.

\subsubsection{Correlation analysis of images before and after encryption}

The degree of correlation between adjacent pixels is directly proportional to its correlation coefficient, and security is inversely proportional. To calculate the correlation between the pixels next to each other of the picture before and after encryption, $N$ pairs of adjacent pixels are stochastically picked to analyze from the three directions of vertical, horizontal and diagonal.

$$
\begin{gathered}
E(x)=\frac{1}{N} \sum_{i=1}^{N} x_{i} \\
D(x)=\frac{1}{N} \sum_{i=1}^{N}\left(x_{i}-E(x)\right)^{2} \\
\operatorname{cov}(x, y)=\frac{1}{N} \sum_{i=1}^{N}\left(x_{i}-E(x)\right)^{2} \\
r_{x y}=\frac{\operatorname{cov}(x, y)}{\sqrt{D(x) D(y)}}
\end{gathered}
$$

where $\operatorname{Cov}(x, y)$ is the correlation function and $D(x)$ means square error. Table 4 shows that the pixels of the encrypted picture are distributed with high dispersion.

Table 4 displayed the related test data. 


\begin{tabular}{|c|c|c|c|c|}
\hline Image & Channel & Horizontal & Vertical & Diagonal \\
\hline \multirow{2}{*}{ Original } & Red & 0.97547 & 0.9874 & 0.96432 \\
image & Green & 0.9762 & 0.98869 & 0.96611 \\
& Blue & 0.95457 & 0.97557 & 0.93619 \\
\hline Encrypted & Red & -0.0036305 & 0.034173 & -0.015494 \\
image & Green & 0.0025112 & 0.030304 & 0.0069924 \\
& Blue & 0.015605 & 0.028112 & 0.005062 \\
\hline
\end{tabular}

\subsubsection{Analysis of resistance to noise}
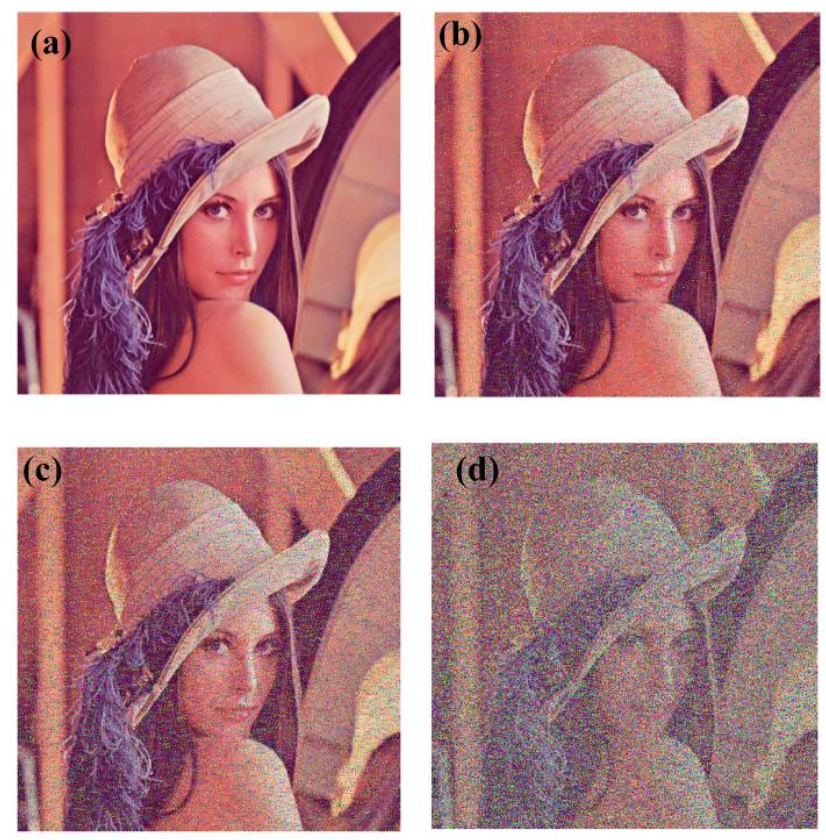

Fig. 18. Decrypted pictures when salt and pepper noise $n$ takes different values: (a) $n$ $=0$, (b) $n=0.05$, (c) $n=0.1$, and (d) $n=0.2$.

In actual communication, when the cipher image is transmitted in the channel, it is inevitably disturbed. One of the most common noises is the salt-and-pepper noise. Figure 18 shows a decrypted image of a ciphered image over the $\mathrm{R}$ channel with different intensities of salt-and-pepper noise. Although there is some distortion in the decoded image, it does not affect the access to valid information. Therefore, the new system resists noise attacks to some extent. 


\section{Conclusion}

In this paper, a class of three-dimensional maps with homogenous multistability and amplitude control is found. A set of united positive Lyapunov exponents is well maintained in attractors with two leaves and three leaves even when they stand at different distances. The complex dynamical properties of the 3-D map are studied using Lyapunov exponents and bifurcation diagrams. The proposed hyperchaotic map can be freely controlled in one dimension or all three dimensions by a single coefficient. These two isolated amplitude controllers provide a quick passage for hyperchaotic sequence rescaling, which is generally a great challenge for typical chaotic systems.

Furthermore, the newly found hyperchaotic map with a sinusoidal function has an infinite number of coexisting attractors with united Lyapunov exponents, in which the special phenomenon is called homogeneous multistability. Experimental results verify the findings from the numerical simulations. A typical application of image encryption is analyzed in detail. Applying the hyperchaotic sequence generated by the map, a color picture can be easily encrypted and decrypted in the key space. The lack of correlation between the histograms and the pixels next to each other proves that the hyperchaotic mapping shows high performance for encryption.

\section{Data availability statement}

The data that support the findings of this study are available from the corresponding author upon reasonable request.

\section{Acknowledgements}

This work was supported financially by the National Natural Science Foundation of China (Grant No.: 61871230), the Natural Science Foundation of Jiangsu Province (Grant No.: BK20181410), and the Provincial Key Training Programs of Innovation and Entrepreneurship for Undergraduates (Grant No.: 201910300053Z). 


\section{Conflict of interest}

We declare that we have no known competing financial interests or personal relationships that could have appeared to influence the work reported in this paper.

\section{References}

[1] F. Yang, J. Mou, J. Liu, Signal processing.: Characteristic analysis of the fractional-order hyperchaotic complex system and its image encryption application. 169, 107373(2020).

[2] G. Peng, F. Min.: Multistability analysis, circuit implementations and application in image encryption of a novel memristive chaotic circuit. Nonlinear Dyn. 90(3), 1607-1625(2017).

[3] Z. Liu, T. Xia, J, Wang.: Image encryption technique based on new two-dimensional fractional-order discrete chaotic map and Menezes-Vanstone elliptic curve cryptosystem Chinese Physics B. 27(3), 030502(2018).

[4] J. Deng, M. Zhou, C. Wang, S. Wang, C. Xu.: Image segmentation encryption algorithm with chaotic sequence generation participated by cipher and multi-feedback loops Multimedia Tools and Applications. 1-20(2021).

[5] J. Zeng, C. Wang, Security and Communication Networks.: A novel hyperchaotic image encryption system based on particle swarm optimization algorithm and cellular automata. 2021(5), 1(2021).

[6] G. Cheng, C. Wang, H. Chen, Int. J. Bifurcat.: A novel color image encryption algorithm based on hyperchaotic system and permutation-diffusion architecture. Chaos. 29(09), 1950115(2019).

[7] Z. Gan, X. Chai, D. Han, Y. Chen.: A chaotic image encryption algorithm based on 3-D bit-plane permutation. Neural Computing and Applications. 31(11), 7111-7130(2019).

[8] X. Chai, X. Fu, Z. Gan, Y. Lu, Y. Chen.: A color image cryptosystem based on dynamic DNA encryption and chaos Signal Processing. 155, 44-62(2019).

[9] Z. Shi, S. Bi, H. Zhang, R. Lu.: Improved auxiliary particle filter - based synchronization of chaotic Colpitts circuit and its application to secure communication. Wireless Communications \& Mobile Computing. 15(10), 1456-1470(2015).

[10] F.H. Min, C. Li, L. Zhang, C.B. Li.: Initial value-related dynamical analysis of the memristor-based system with reduced dimensions and its chaotic synchronization via adaptive sliding mode control method. Chinese Journal of Physics. 58, 117-131(2019).

[11] J. Liu, S. Liu, C. Yuan.: Adaptive complex modified projective synchronization of complex chaotic (hyperchaotic) systems with uncertain complex parameters. Nonlinear Dynamics. 79(2), 1035-1047(2015).

[12] J. Liu, S. Liu, J.C. Sprott.: Adaptive complex modified hybrid function projective synchronization of different dimensional complex chaos with uncertain complex parameters. Nonlinear Dynamics. 83(1), 1109-1121(2016).

[13] L.P. Zhang, Y. Liu, Z.C. Wei, H.B. Jiang.: A novel class of two-dimensional chaotic maps with infinitely many coexisting attractors. Chinese Physics B. 29(6), 060501(2020).

[14] B.C. Bao, H.Z. Li, L. Zhu, X. Zhang.: Initial-switched boosting bifurcations in 2D hyperchaotic map. Chaos. 30(3), 033107(2020).

[15] H. Li, H. Bao, L. Zhu, B. Bao, M. Chen.: Extreme Multistability in Simple Area-Preserving Map. IEEE Access. 8, 175972-175980(2020).

[16] C. Li, J.C. Sprott.: Finding coexisting attractors using amplitude control. Nonlinear Dynamics. 73(3), 1335-1341(2013). 
[17] C. Li, J.C. Sprott, A. Akgul, H.H.C. lu.: A new chaotic oscillator with free control. Chaos. 27(8), 083101(2017).

[18] C. Li, X. Wang, G. Chen.: Diagnosing multistability by offset boosting. Nonlinear Dynamics. 90(2), 1335 - 1341(2017).

[19] C. Li, J.C. Sprott.: Coexisting hidden attractors in a 4-D simplified Lorenz system. International Journal of Bifurcation and Chaos. 24(03), 1450034(2014).

[20] H. Li, H. Bao, L. Zhou, B. Bao, M. Chen.: Extreme Multistability in Simple Area-Preserving Map. IEEE Access. 8, 175972 - 175980(2020).

[21] A.N. Pisarchik, U. Feudel.: Control of multistability. Physics Reports. 540(4), 167-218(2014).

[22] X. Han, F. Xia, C. Zhang, Y. Yu.: Origin of mixed-mode oscillations through speed escape of attractors in a Rayleigh equation with multiple-frequency excitations. Nonlinear Dynamics. 88(4), 2693-2703(2017).

[23] W. Zhou, G. Wang, Y. Shen, F. Int. J. Bifurcat.: Hidden coexisting attractors in a chaotic system without equilibrium point. Chaos. 28(10), 1830033(2018).

[24] V.T. Pham, C. Volos, S. Jafari, T. Kapitaniak.: Coexistence of hidden chaotic attractors in a novel no-equilibrium system. Nonlinear Dynamics. 87(3), 2001-2010(2017).

[25] O.S. Ojoniyi, A.N. Njah.: A 5D hyperchaotic Sprott B system with coexisting hidden attractors. Chaos, Solitons \& Fractals. 87, 172-181(2016).

[26] L. Xu, Z. Li, J. Li, W. Hua.: A novel bit-level image encryption algorithm based on chaotic maps. Optics and Lasers in Engineering. 78, 17-25(2016).

[27] H. Bao, Z. Hua, N. Wang, L. Zhu.: Initials-Boosted Coexisting Chaos in a 2-D Sine Map and Its Hardware Implementation. IEEE Transactions on Industrial Informatics. 17(2), 1132-1140(2020).

[28] F. Hadjabi, A. Ouannas, N. Shawagfeh, A.A. Khennaoui.: On two-dimensional fractional chaotic maps with symmetries. Symmetry. 12(5), 756(2020).

[29] H. Huang, S. Yang, R. Ye.: Chaotic image encryption based on bidimensional empirical mode decomposition and double random phase encoding. IET Image Processing. 79(37), 28065-28078(2020).

[30] S. Zhang, Y. Zeng, Z. Li, C. Zhou.: Hidden extreme multistability, antimonotonicity and offset boosting control in a novel fractional-order hyperchaotic system without equilibrium. Int. J. Bifurcat. Chaos. 28(13), 1850167(2018).

[31] C. Li, J. Sun, T. Lu, J.C. Sprott, Z. Liu.: Polarity balance for attractor self-reproducing. Int. J. Bifurcat. Chaos. 30(6), 063144(2020).

[32] F. Yuan, Y. Jin, Y. Li.: Self-reproducing chaos and bursting oscillation analysis in a meminductor-based conservative system. Int. J. Nonlinear Science. Chaos. 30(5), 053127(2020).

[33] J. Gu, C. Li, Y. Chen, H.H.C. lu, T. Lei.: A conditional symmetric memristive system with infinitely many chaotic attractors. IEEE Access. 8, 12394-12401(2020).

[34] P.V. Kupysov, S.P. Kuznetsov, Int. J.: Route to hyperbolic hyperchaos in a nonautonomous time-delay system. Nonlinear Science. Chaos. 30(11), 113113(2020). 
Figures
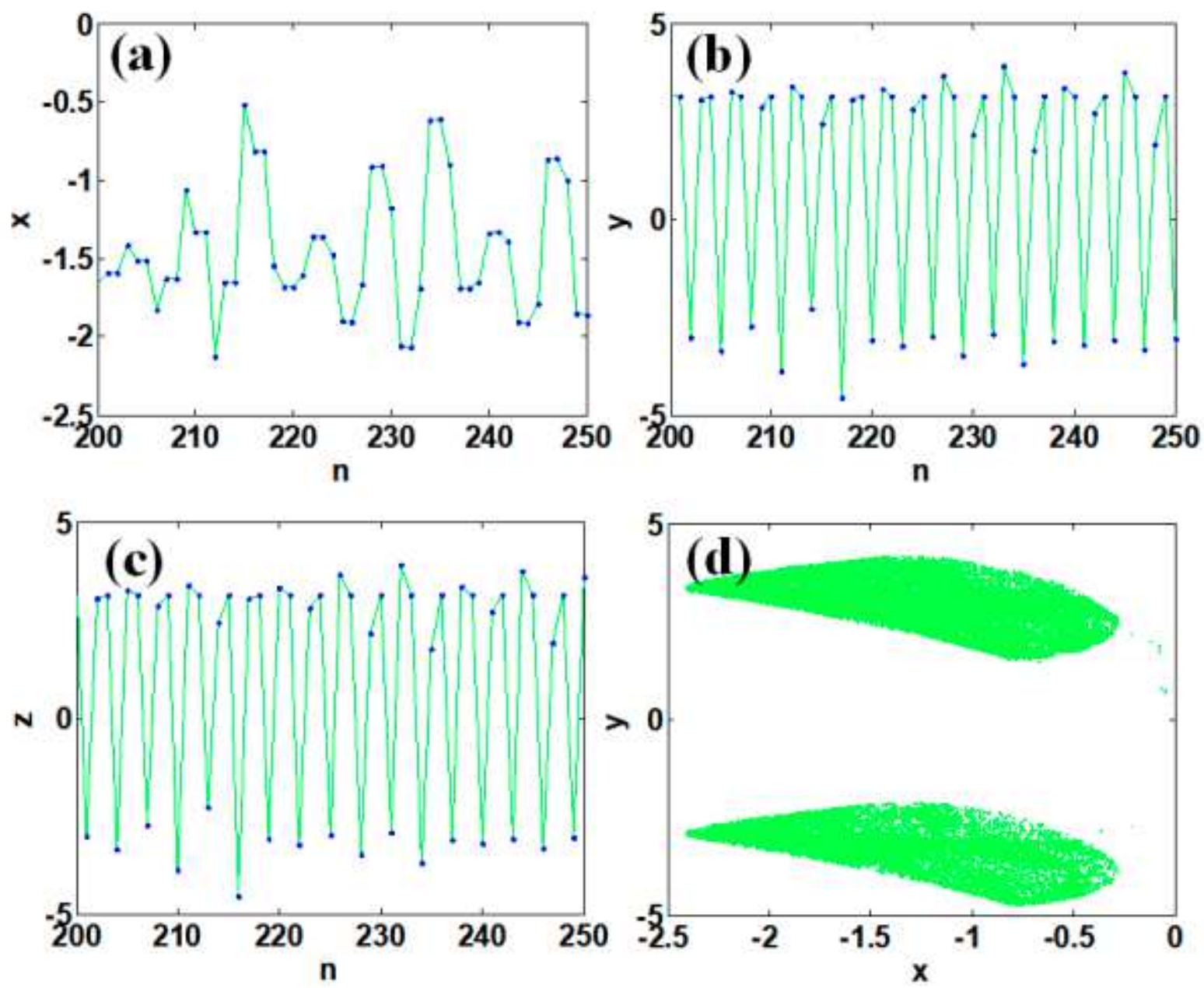

Figure 1

Please see the Manuscript PDF file for the complete figure caption
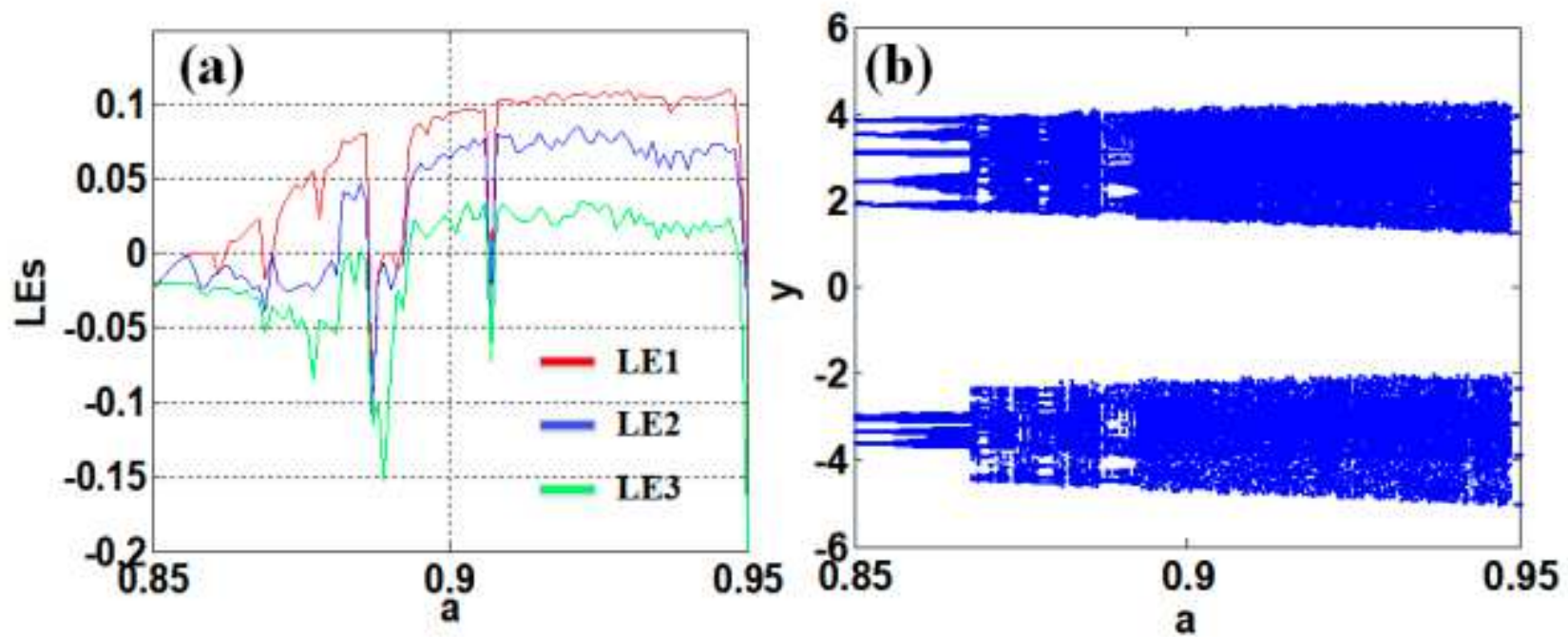
Figure 2

Please see the Manuscript PDF file for the complete figure caption
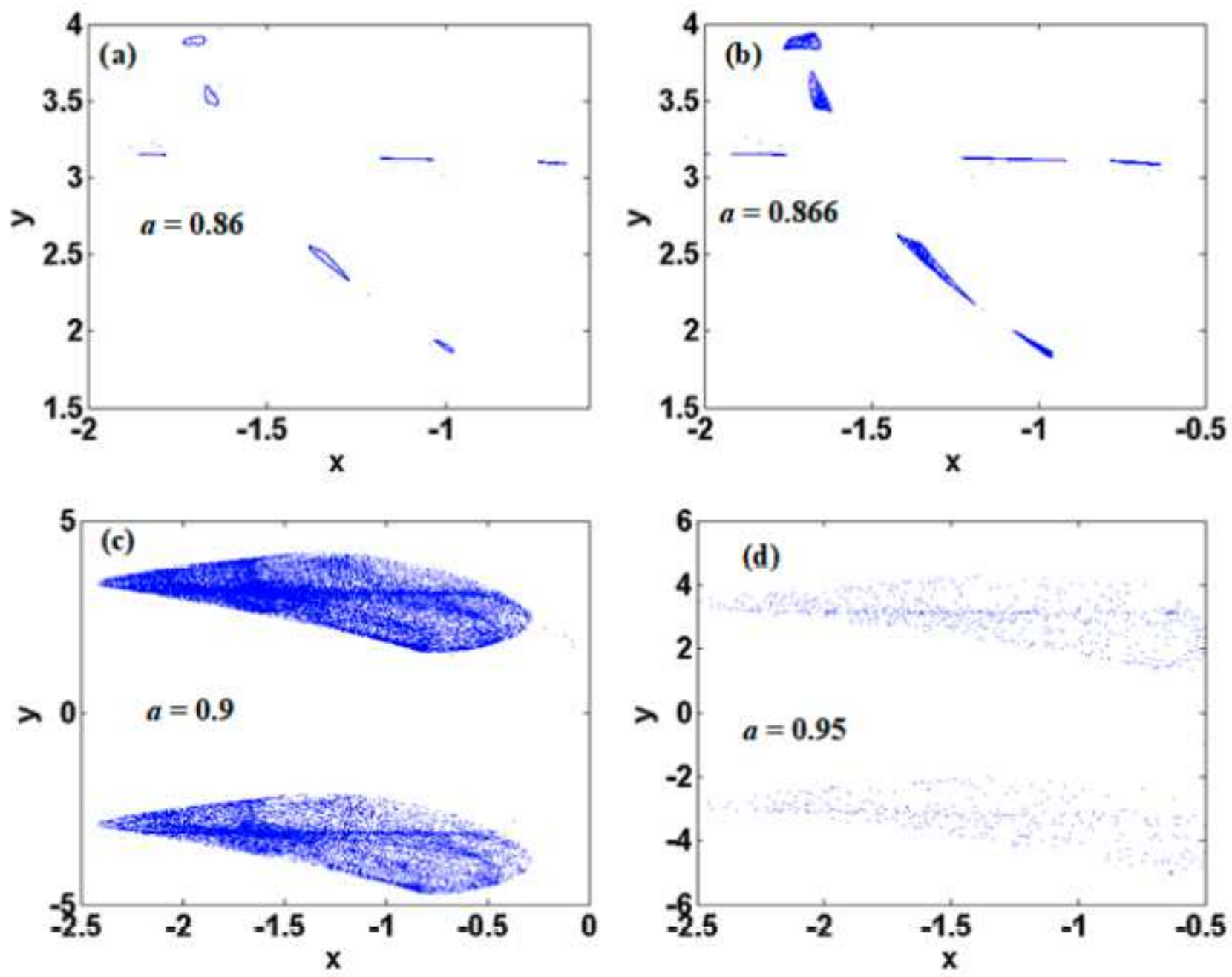

Figure 3

Please see the Manuscript PDF file for the complete figure caption
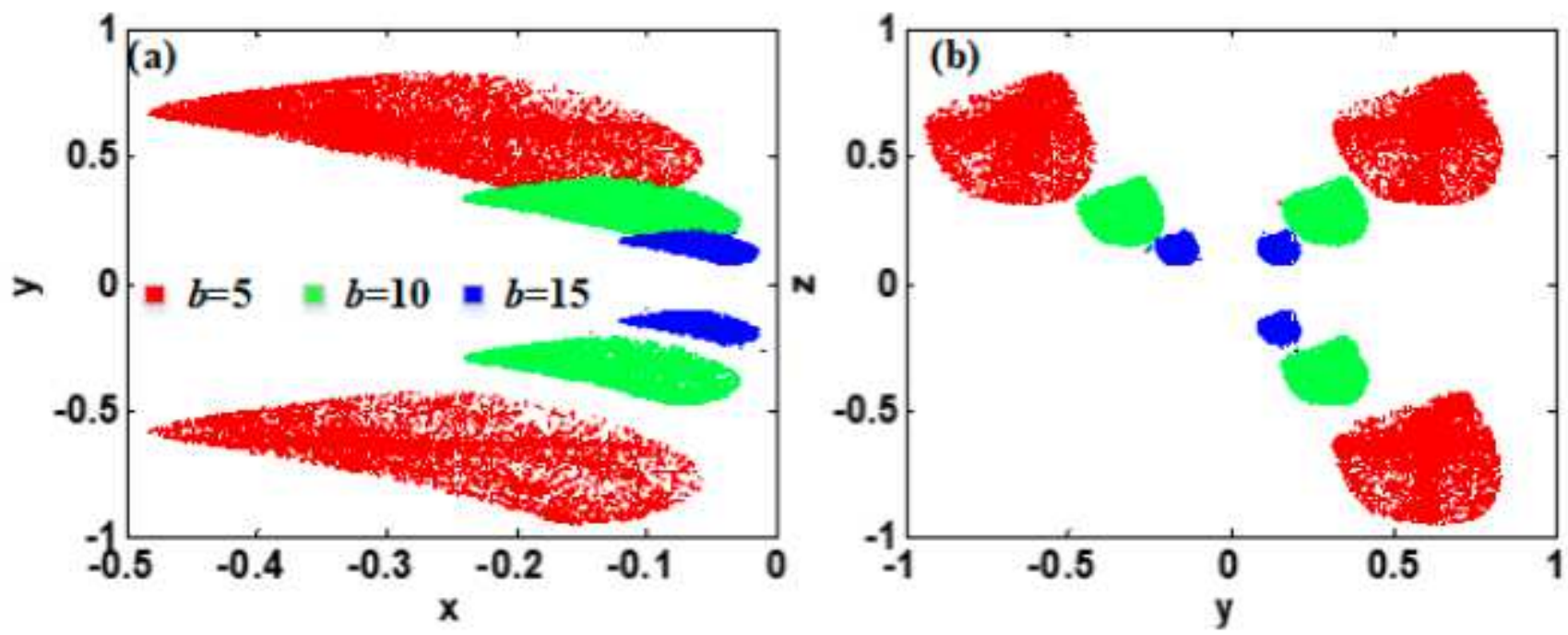
Figure 4

Please see the Manuscript PDF file for the complete figure caption
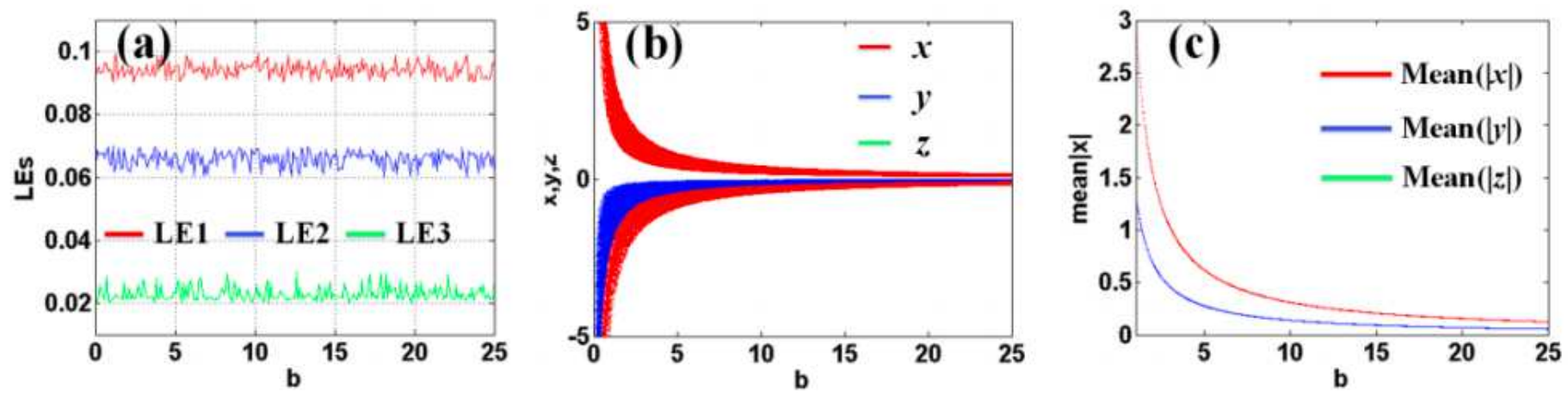

Figure 5

Please see the Manuscript PDF file for the complete figure caption
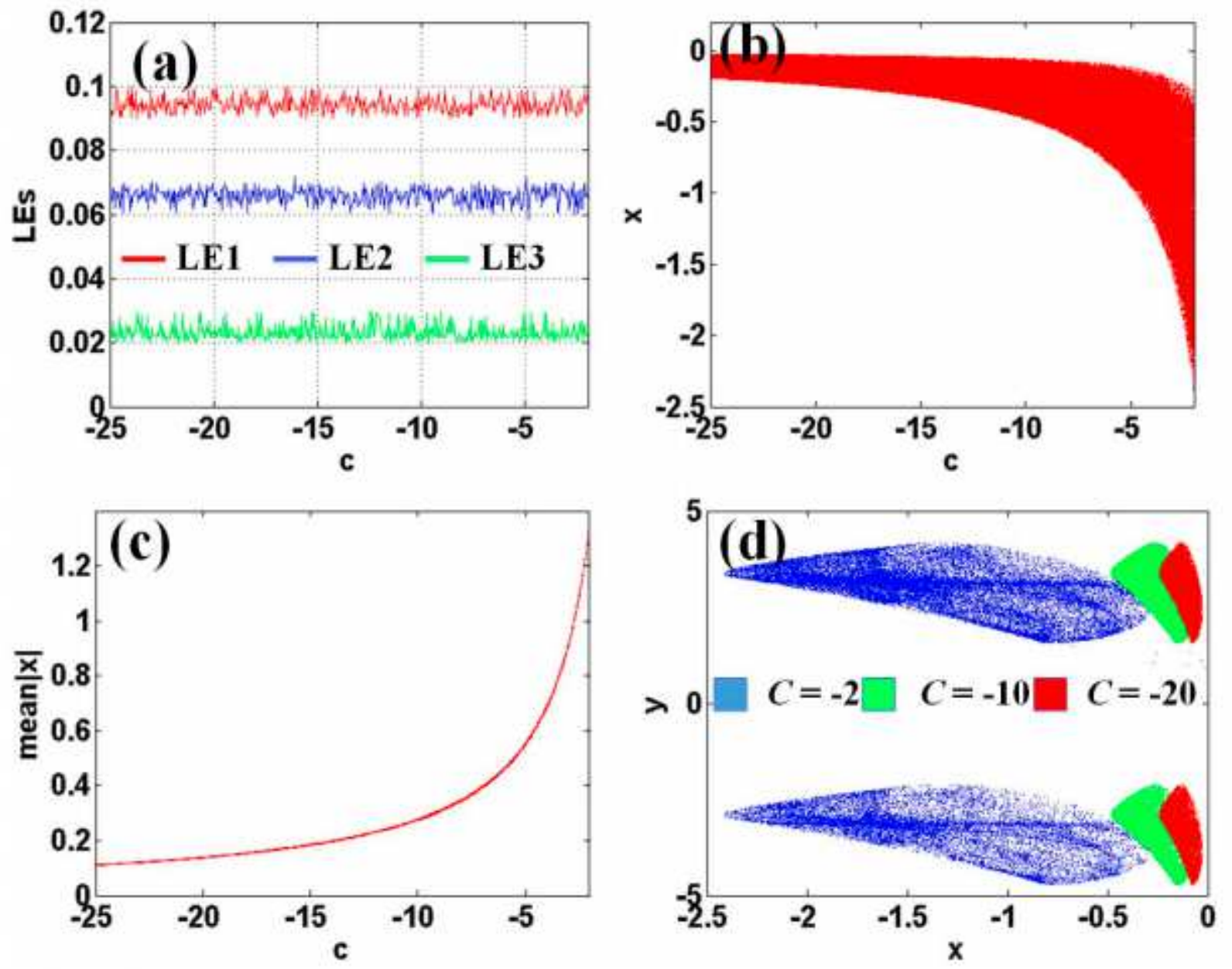

Figure 6

Please see the Manuscript PDF file for the complete figure caption 

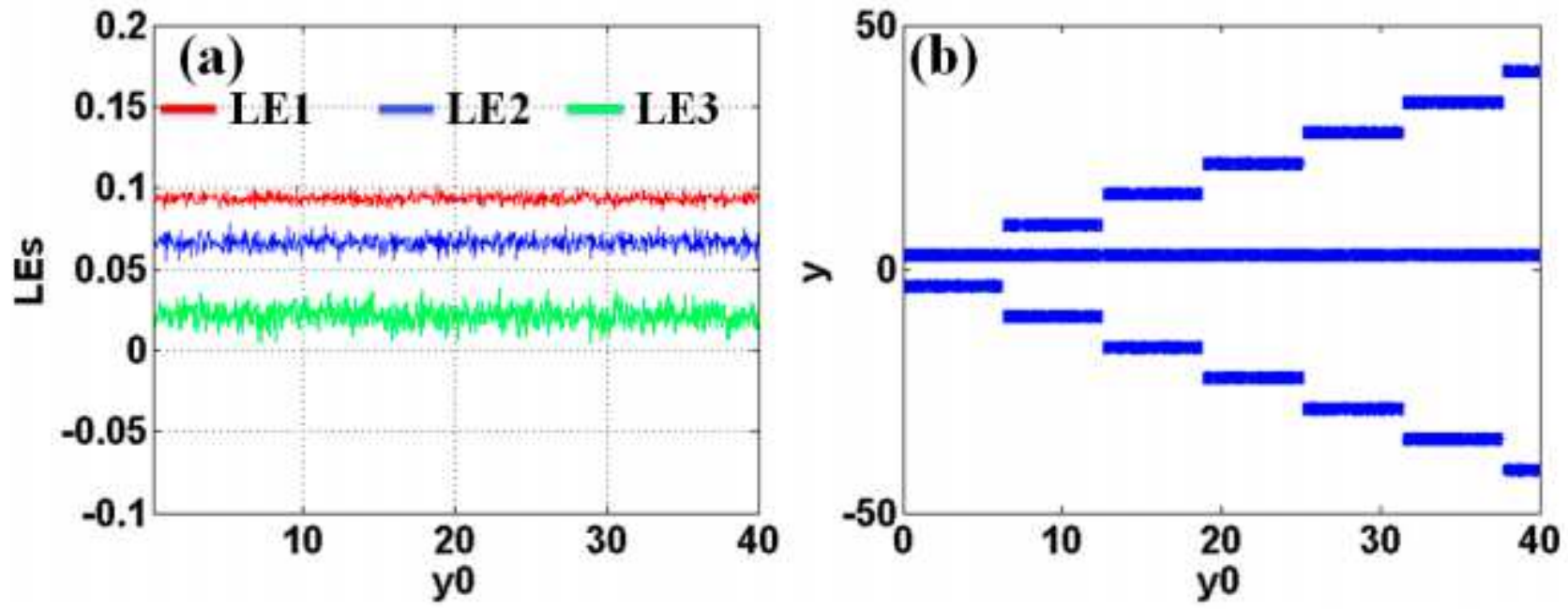

Figure 7

Please see the Manuscript PDF file for the complete figure caption 

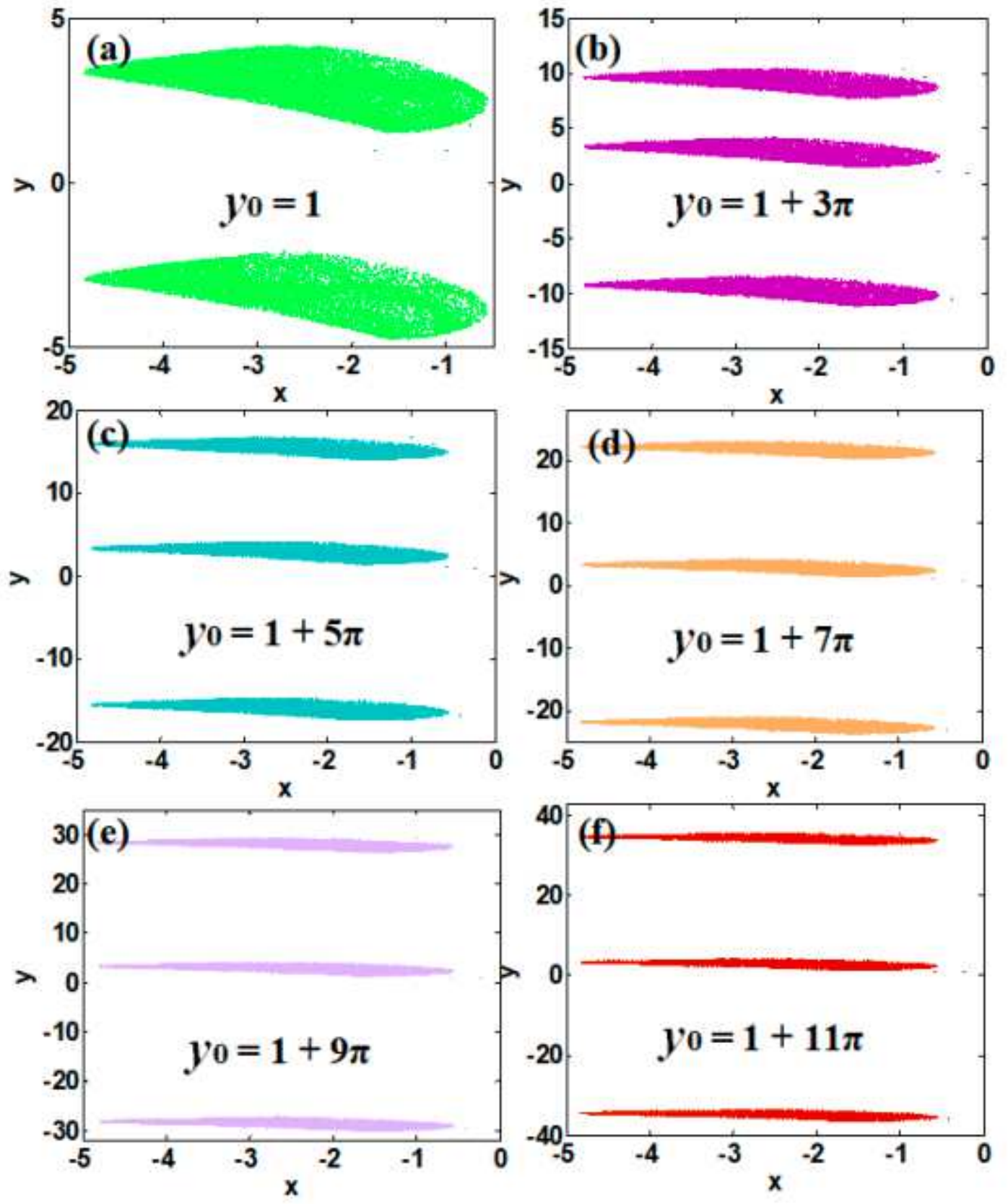

Figure 8

Please see the Manuscript PDF file for the complete figure caption 

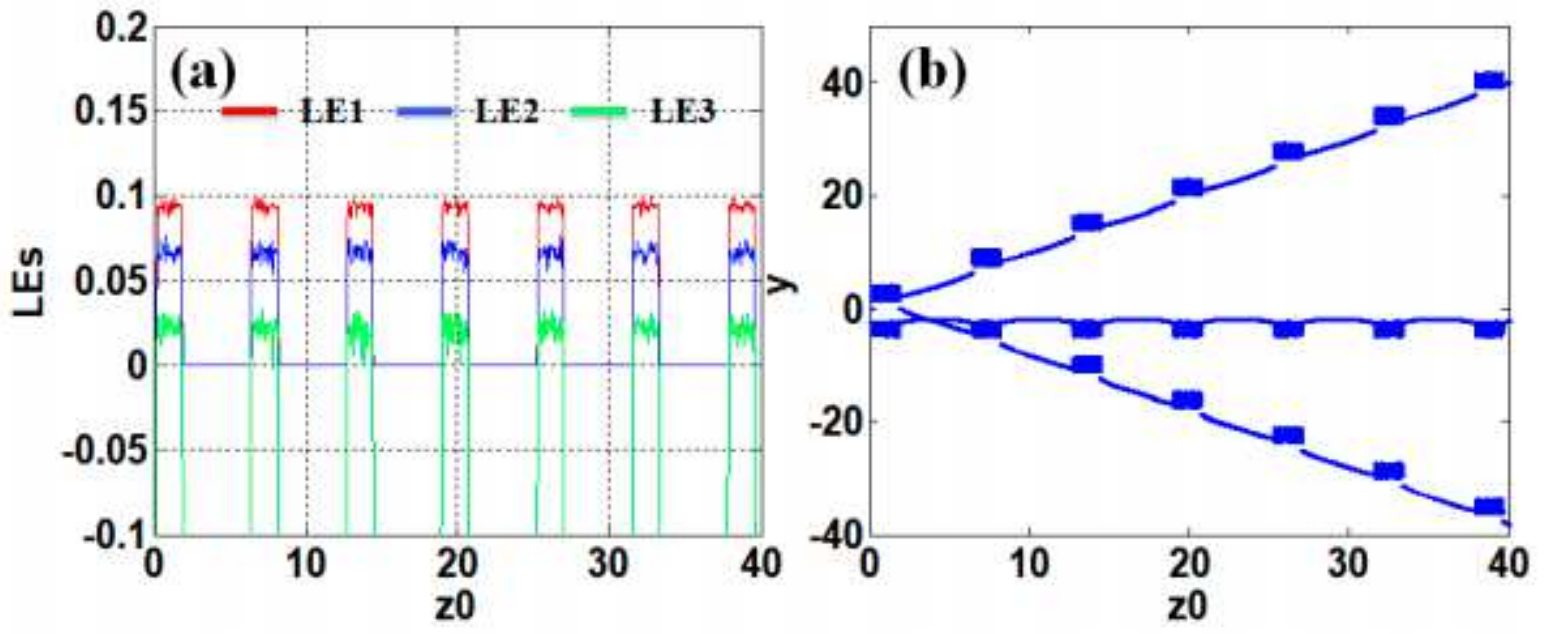

Figure 9

Please see the Manuscript PDF file for the complete figure caption 

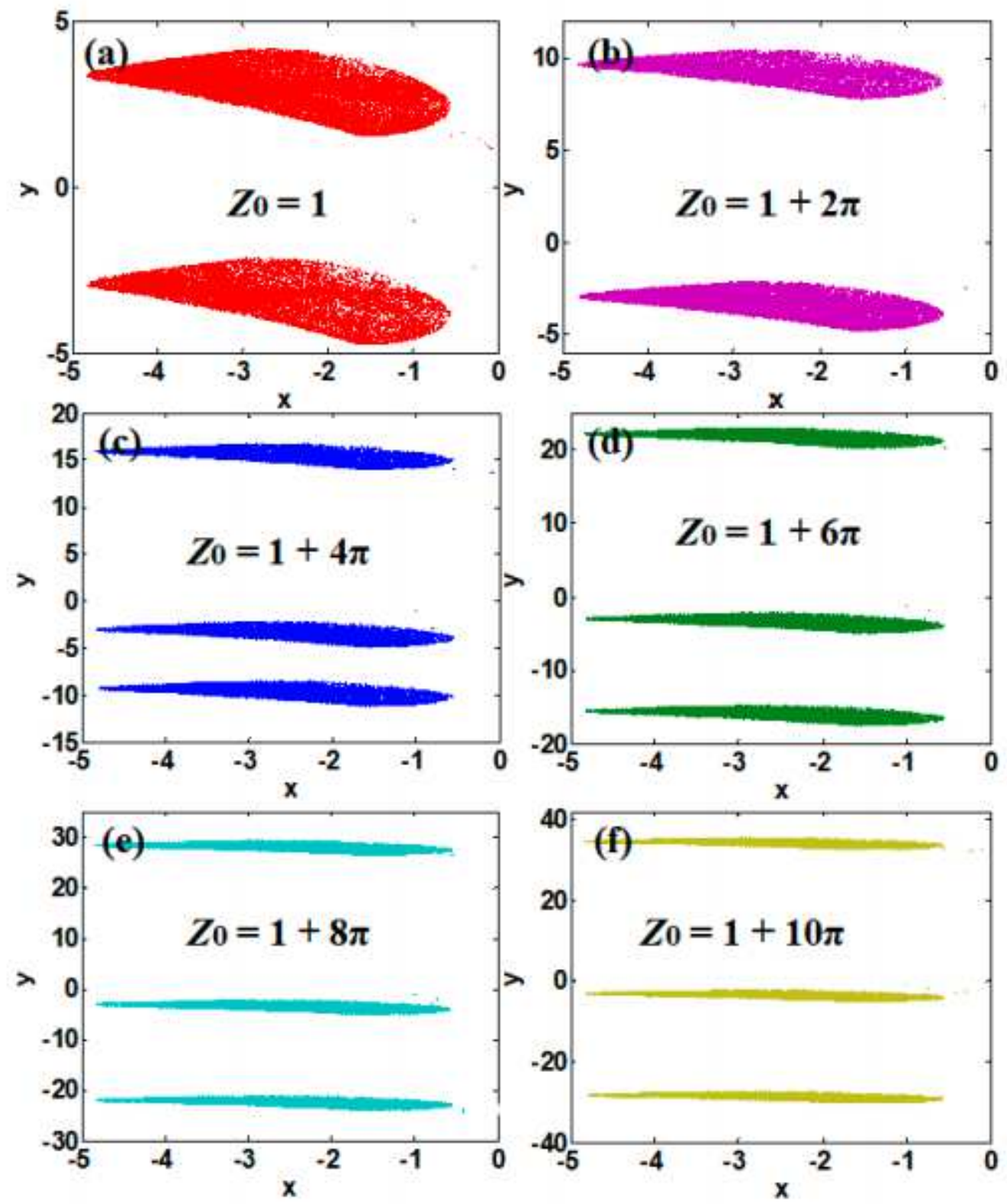

Figure 10

Please see the Manuscript PDF file for the complete figure caption
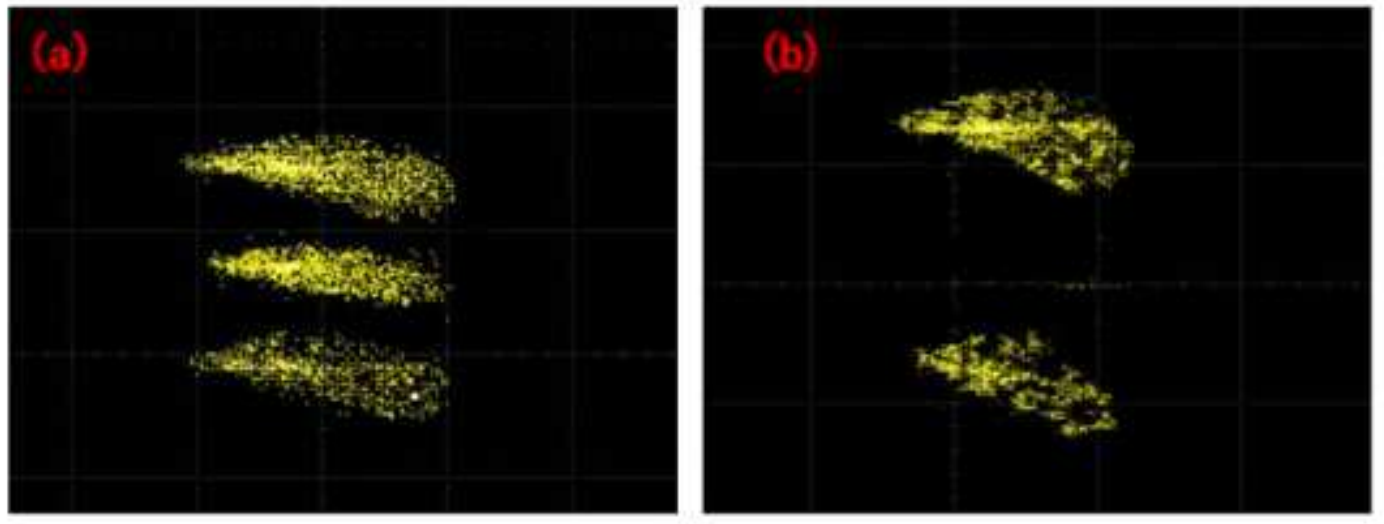


\section{Figure 11}

Please see the Manuscript PDF file for the complete figure caption

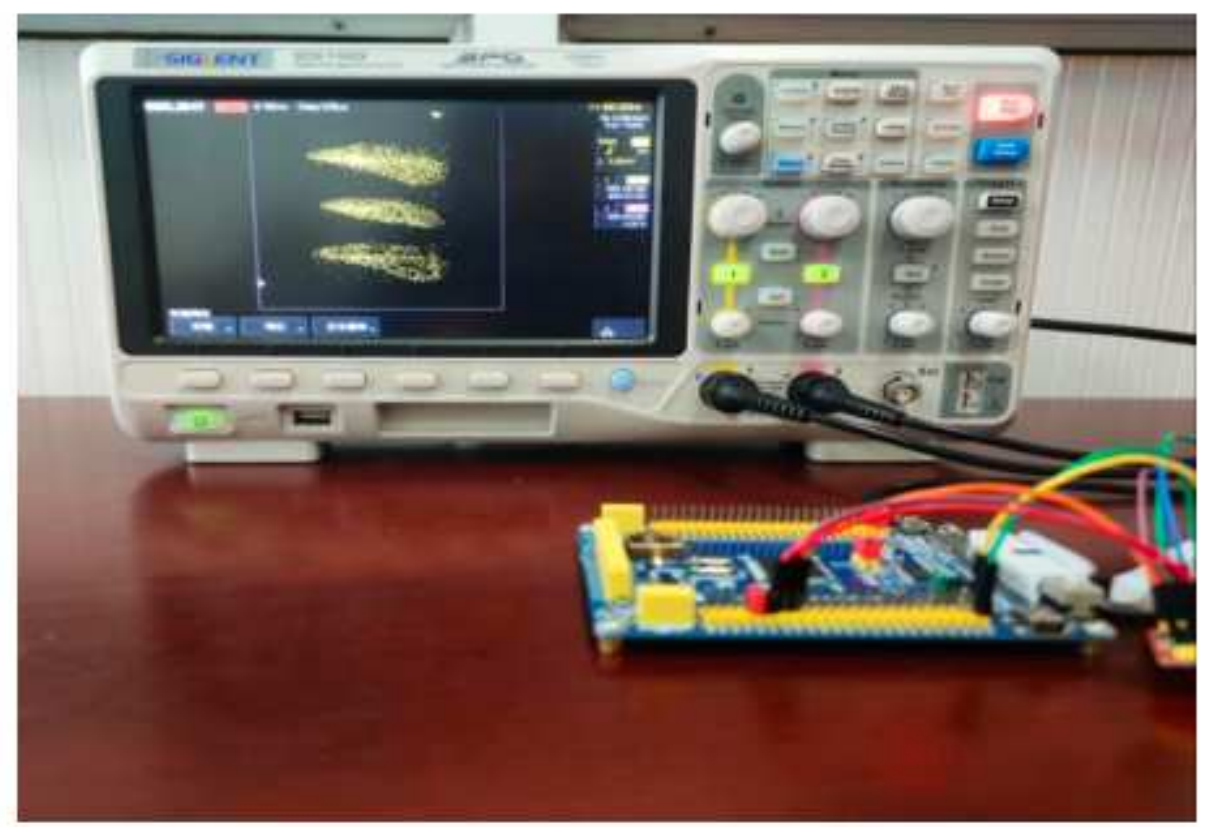

Figure 12

Please see the Manuscript PDF file for the complete figure caption

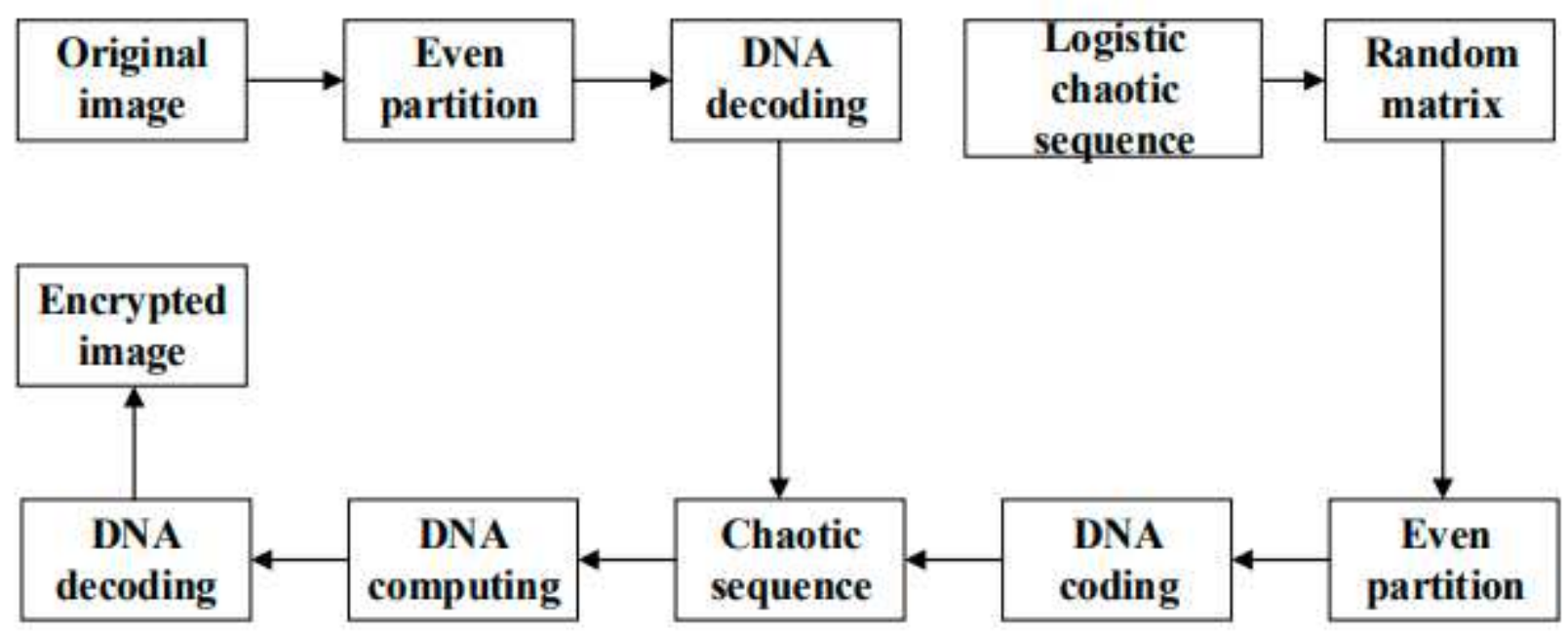

Figure 13

Please see the Manuscript PDF file for the complete figure caption 

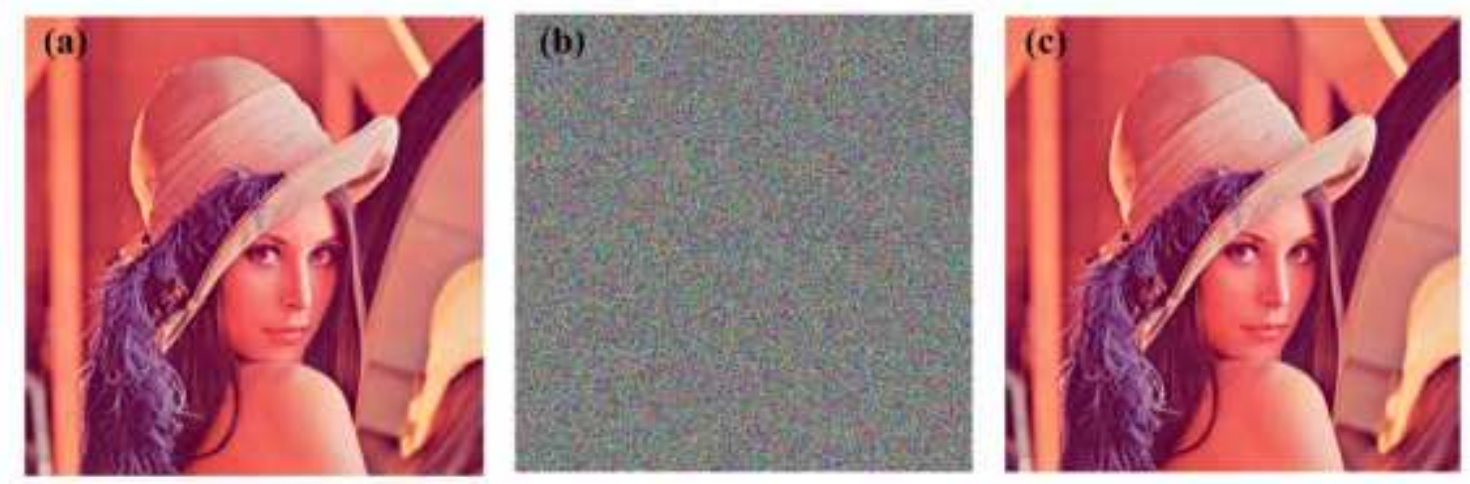

\section{Figure 14}

Please see the Manuscript PDF file for the complete figure caption
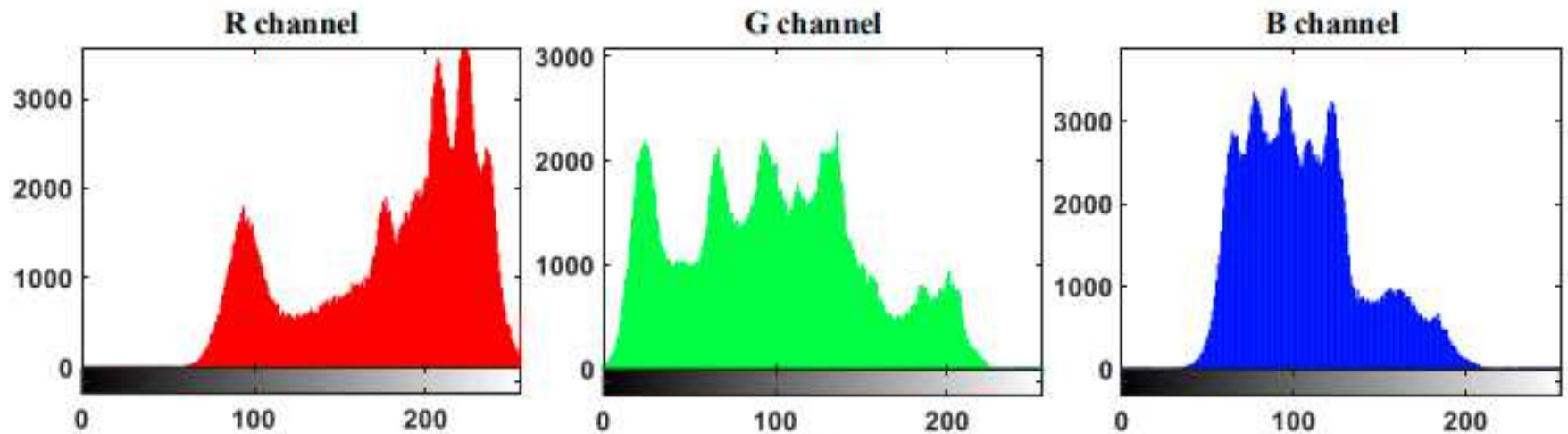

(a)
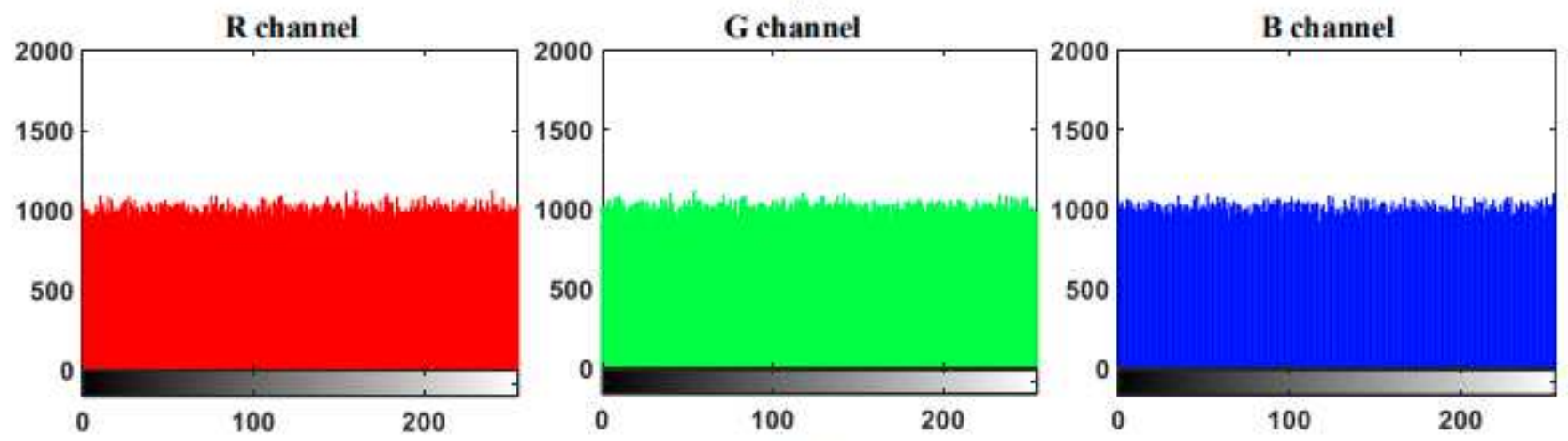

(b)

\section{Figure 15}

Please see the Manuscript PDF file for the complete figure caption 


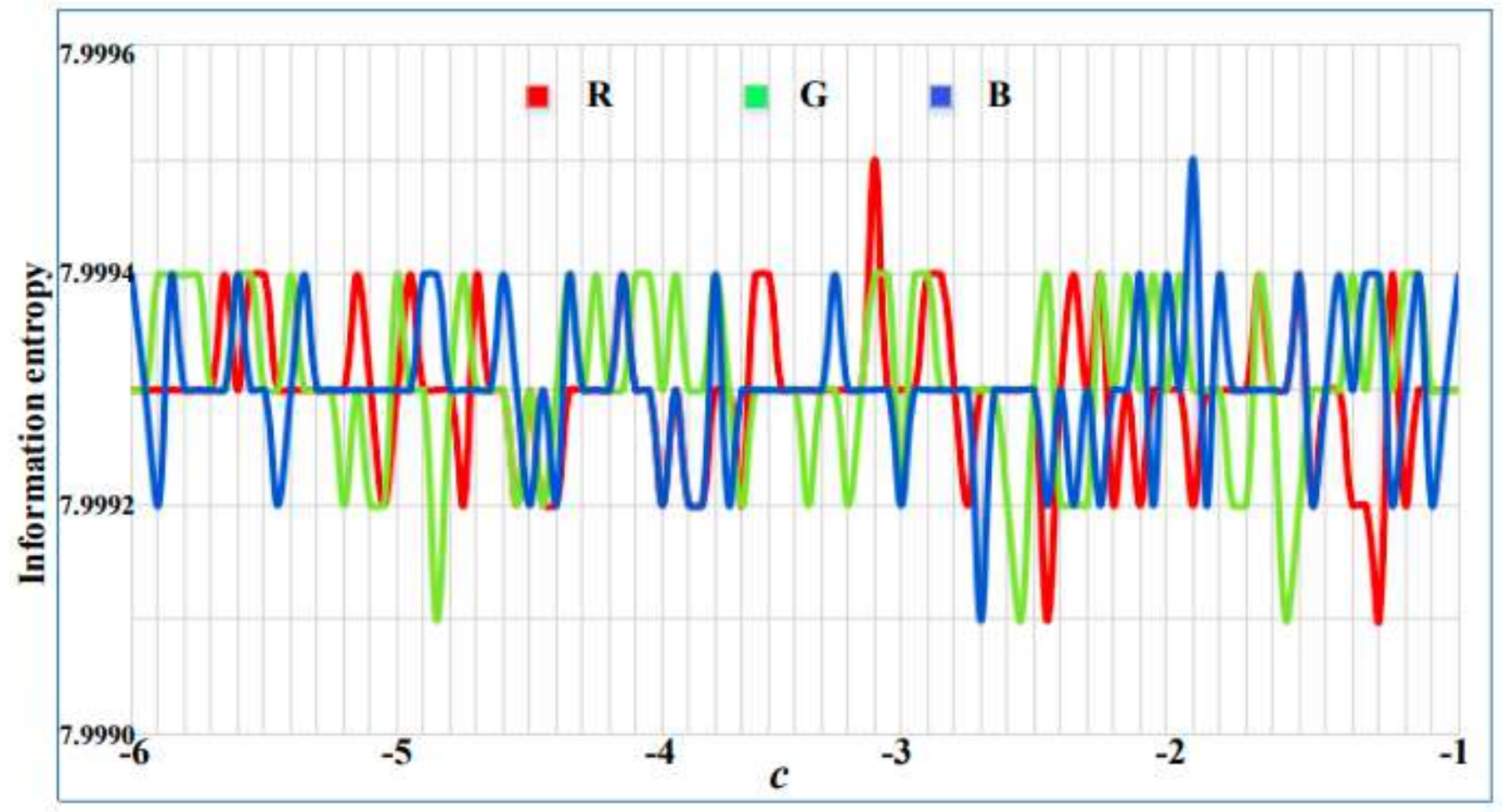

Figure 16

Please see the Manuscript PDF file for the complete figure caption

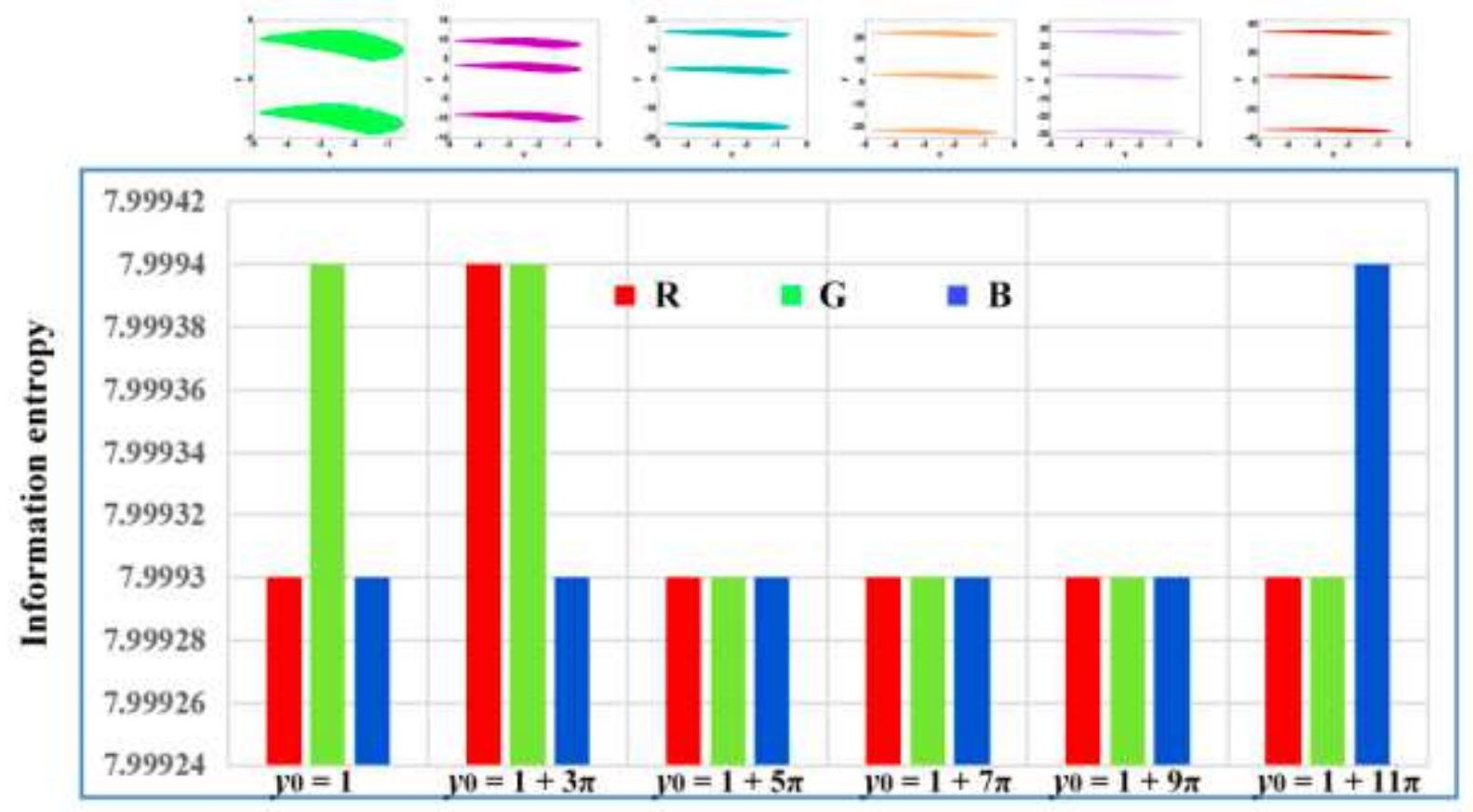

Figure 17

Please see the Manuscript PDF file for the complete figure caption 

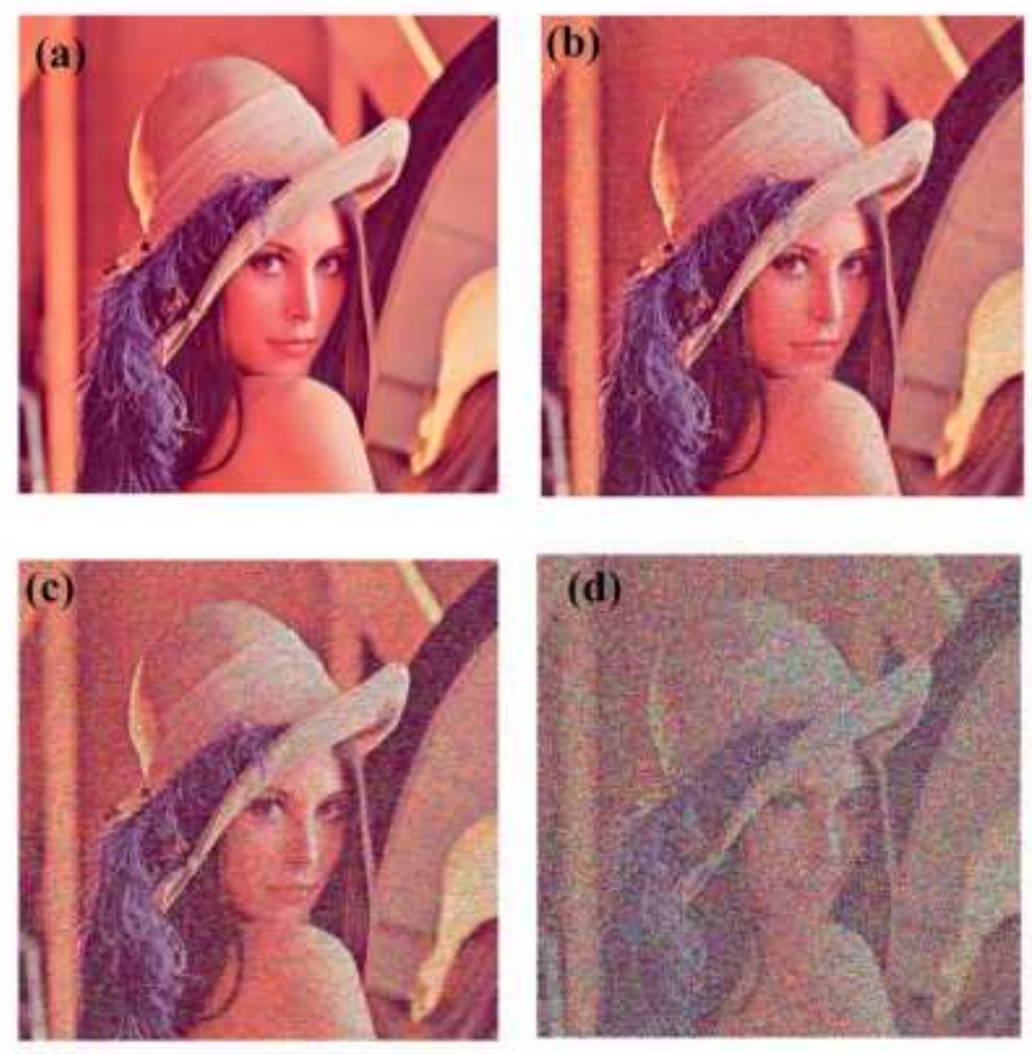

Figure 18

Please see the Manuscript PDF file for the complete figure caption 